\title{
Mesoscale simulations of two model systems in biophysics: from red blood cells to DNAs
}

\author{
Zhangli Peng $^{1} \cdot$ Yeng-Long Chen ${ }^{2} \cdot$ Huijie Lu ${ }^{1} \cdot$ Zehao Pan $^{3} \cdot$ Hsueh-Chia Chang ${ }^{3}$
}

Received: 17 January 2015 / Revised: 10 May 2015 / Accepted: 25 June 2015 / Published online: 10 September 2015 (C) OWZ 2015

\begin{abstract}
Computational modeling has become increasingly important in biophysics, but the great challenge in numerical simulations due to the multiscale feature of biological systems limits the capability of modeling in making discoveries in biology. Innovative multiscale modeling approaches are desired to bridge different scales from nucleic acids and proteins to cells and tissues. Although all-atom molecular dynamics has been successfully applied in many microscale biological processes such as protein folding, it is still prohibitively expensive for studying macroscale problems such as biophysics of cells and tissues. On the other hand, continuum-based modeling has become a mature procedure for analysis and design in many engineering fields, but new insights for biological systems in the microscale are limited when molecular details are missing in continuum-based modeling. In this context, mesoscale modeling approaches such as Langevin dynamics, lattice Boltzmann method, and dissipative particle dynamics have become popular by simultaneously incorporating molecular interactions and long-range hydrodynamic interactions, providing insights to properties on longer time and length scales than molecular dynamics. In this review, we summarized several mesoscale simulation approaches for studying two model systems in biophysics: red blood cells (RBCs) and deoxyribonucleic acids (DNAs). The RBC is a model system for cell mechanics and biological membranes, while the DNA represents a model system for
\end{abstract}

\footnotetext{
Zhangli Peng

zpeng3@nd.edu

1 Department of Aerospace and Mechanical Engineering, University of Notre Dame, Notre Dame, USA

2 Institute of Physics, Academia Sinica, Taipei, Taiwan

3 Department of Chemical and Biomolecular Engineering, University of Notre Dame, Notre Dame, USA
}

biopolymers. We introduced the motivations of studying these problems and presented the key features of different mesoscale methods. Furthermore, we described the latest progresses in these methods and highlighted the major findings for modeling RBCs and DNAs. Finally, we also discussed the challenges and potential issues of different approaches.

Keywords Erythrocyte $\cdot$ Translocation $\cdot$ Nanopore $\cdot$ Lipid bilayer $\cdot$ Cytoskeleton $\cdot$ Microfluidics $\cdot$ Sequencing

\section{Introduction}

Along with experimental and theoretical investigations, computational modeling has become increasingly important in the fields of biomechanics and biophysics [1], thank to the following three facts. First, the speed of computation has been increased tremendously in the past decades due to the innovations of CPUs and parallel computing architecture, following the observations of Moore's law. Supercomputers with special purposes for carrying out molecular dynamics (MD) simulations, such as Anton [2], have also been developed to enable the study of protein folding for milliseconds. Secondly, the detailed structures of proteins and cells have been extensively characterized using experimental techniques such as crystallography and super-resolution electron microscopes [3]. These characterizations have allowed us to build accurate computational models based on experimental measured topology and geometry. In addition, physical properties such as molecular stiffness and entropic elasticity have also been measured using state-of-the-art tools such as AFM and optical tweezers [4], so that they can be incorporated into the computational models. Thirdly, computational methods have also been advanced significantly along with the hard- 
ware development, such as the developments of multiscale modeling approaches $[5,6]$. However, in many cases of biological studies, it is still challenging to rely on computational modeling to make discoveries and to guide design. The current main bottle neck is the lack of efficient computational methods to address the large separation of spatial and temporal scales between the properties of single molecules and those of bulk materials.

Continuum-based modeling such as finite element method (FEM) [7,8], boundary element method (BEM) [9], finite difference method (FDM) [10], finite volume method (FVM) [11], and spectral element method (SEM) [12] have been successfully applied to study biomechanical and biophysical problems for organs, tissues, and cells [13-15]. These methods have been used extensively in different engineering fields and have become the mature and routine procedures, with commercial packages available for designing buildings, bridges, and automobiles [8]. However, due to the multiscale and heterogeneous nature of biological systems, it is often difficult to gain new insights with only continuum-based models. The problem is particularly severe for small systems such as the cell membrane and biopolymers in which thermal fluctuations strongly influence measurements and molecular properties.

On the other hand, MD has been employed to resolve many significant problems in small length scales, such as protein folding $[5,16]$. However, the length and time scales accessible to MD are currently limited to nanometers in length and microseconds in time. Even with specially designed supercomputer [2], it can only reach millisecond to simulate proteins without complicated hierarchical structures. Although all-atom MD is able to provide new insights for problems with short characteristic lengths and times, many important biological processes for larger bio-molecules and cells that occur over seconds or even minutes are expected to be beyond all-atom MD for the next decade or longer.

To deal with various complex multiscale biological systems, a good starting point is to study some 'model' systems as examples. Red blood cells (RBCs) and deoxyribonucleic acids (DNAs) are good examples of biological systems with multiple characteristic lengths and times. The RBC is a model system for cell mechanics and biological membranes, while the DNA is a model system for biopolymers. Multiscale studies of RBCs span from the properties of membrane proteins to whole cell deformation. Several known protein mutations in the RBC membrane can result in altered mechanical properties of RBCs, leading to diseases such as hereditary spherocytosis [17]. For DNAs, the length scales span from the nucleobase $(\sim 1 \AA)$ to the contour length (larger than 1 meter for the human genome DNA). The large variation in these characteristic lengths presents a severe challenge for bridging between continuum-based and atomistic-based models. Recently, several mesoscale modeling approaches emerged rapidly as ways to study these problems in which it is forbidden to use all-atom MD to simulate the entire system, while continuum-based modeling cannot reveal the mechanisms related to molecular details $[18,19]$. In this review, we will briefly describe different mesoscale models for studying RBCs and DNAs.

\section{Mesoscale modeling of red blood cell dynamics}

Due to its simple membrane structure, red blood cells (RBCs, erythrocytes) have been studied as a model system for cell biomechanics [13,14]. A RBC has no nucleus or other important organelle. It consists of hemoglobin solution and a biological composite membrane including a lipid bilayer and a cortex cytoskeleton [33]. There are about 33,000 Junctional Complexes (JCs) in the cytoskeleton, which is a basic protein unit, consisting of an actin protofilament connected by four to fix spectrin proteins. The cytoskeleton is attached to the lipid bilayer through the transmembrane proteins such as band 3 and glycophorin C. Furthermore, the transmembrane proteins can move freely in the lipid bilayer so that the cytoskeleton can slide against the lipid bilayer. The interaction between the lipid bilayer and the cytoskeleton is an important problem since it is related to physiological process such as RBC aging [34] and pathological mechanisms such as bilayer loss in hereditary spherocytosis [17], but this bilayer-cytoskeletal interaction remains unquantified due to its small length scale. It has been found that bilayer-cytoskeletal interactions affect RBC membrane fluctuations [19] and tank-treading motion in shear flow [35]. For example, different bilayercytoskeletal tangential friction coefficients in diseased and healthy RBCs can lead to different cytoskeleton density distributions and different bilayer-cytoskeletal interaction forces in shear flow [35]. In addition, only by accounting for bilayer-cytoskeletal vertical elastic interaction, the magnitude of membrane fluctuations can be predicted [19]. However, the RBC membrane is considered as a single continuum layer in most existing models in the literature [3639]. Recently continuum-based and particle-based mesoscale models have been developed to consider different molecular details of the RBC membrane [20-25,27-32,35], as shown in Fig. 1. In the following, we will review three different mesoscale approaches as examples, including a three-level hierarchical multiscale continuum approach, a two-component dissipative particle dynamics approach and a two-component coarse-grained MD approach.

\subsection{Two-component RBC model using three-level hierarchical multiscale continuum method}

Peng et al. have developed a three-level multiscale model of RBCs and coupled it with a boundary element model to 


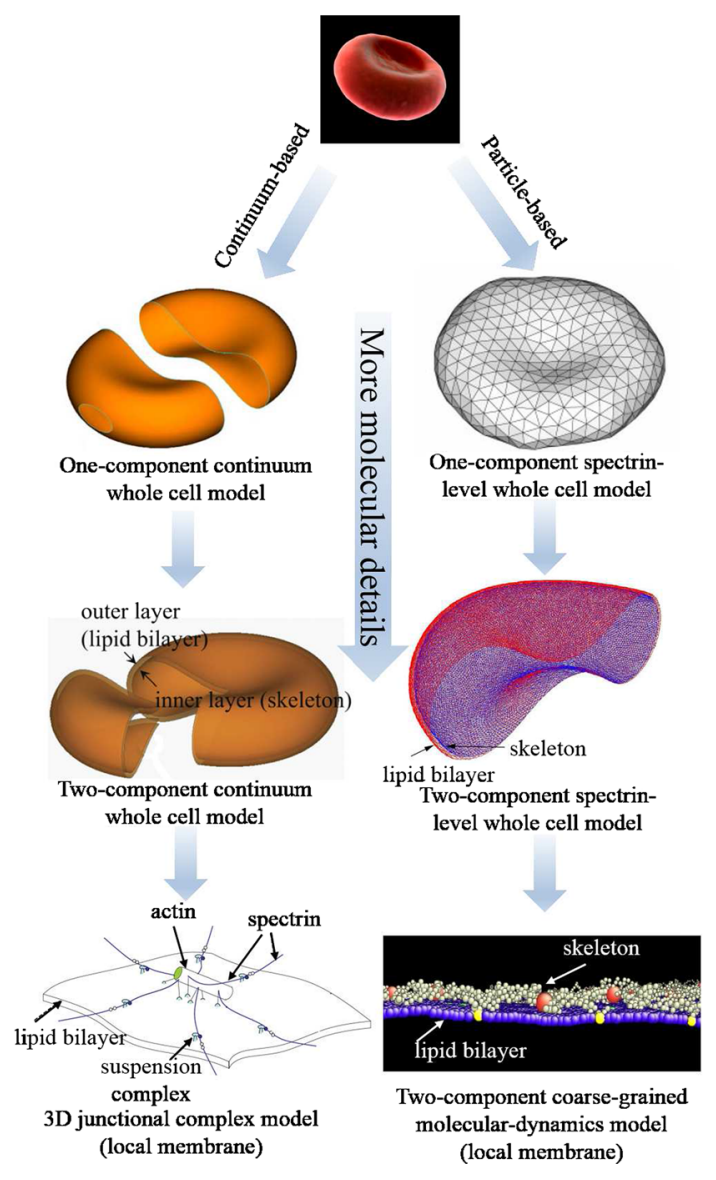

Fig. 1 Continuum-based and particle-based (also called, atomisticbased) RBC models with different molecular details: One-component continuum whole cell model [20], two-component continuum whole cell model $[21,22]$, one-component spectrin-level whole cell model [23-25], two-component spectrin-level whole cell MODEL [26,27], 3D junctional complex (JCs) model [28,29], and two-component coarsegrained molecular-dynamics model [30-32]

study the dynamic response of RBCs in tube and shear flows [21]. For the RBC membrane, we built a three-level multiscale model to simulate its viscoelastic behaviors at different length scales, including single protein scale, protein complex scale and cell scale. For the fluids surrounding the RBC, we used a boundary element approach to study the fluid dynamics based on the Stokes equation of viscous flow [22].

The dynamics of RBCs involves physics at different length scales. For example, in the whole cell level, RBCs may undergo tank-treading motions in shear flow in the micro meter scale $[40,41]$. In the protein complex scale, JCs of RBCs may experience fluctuations due to mode switching $[28,42]$. In the protein level, tension may induce spectrin unfolding in the nanometer scale $[28,43]$. We developed a multiscale framework to investigate the physics in different length scales, including three models at three different length scales, and coupled them together using a hierarchical multiscale approach, as shown in Fig. 2.

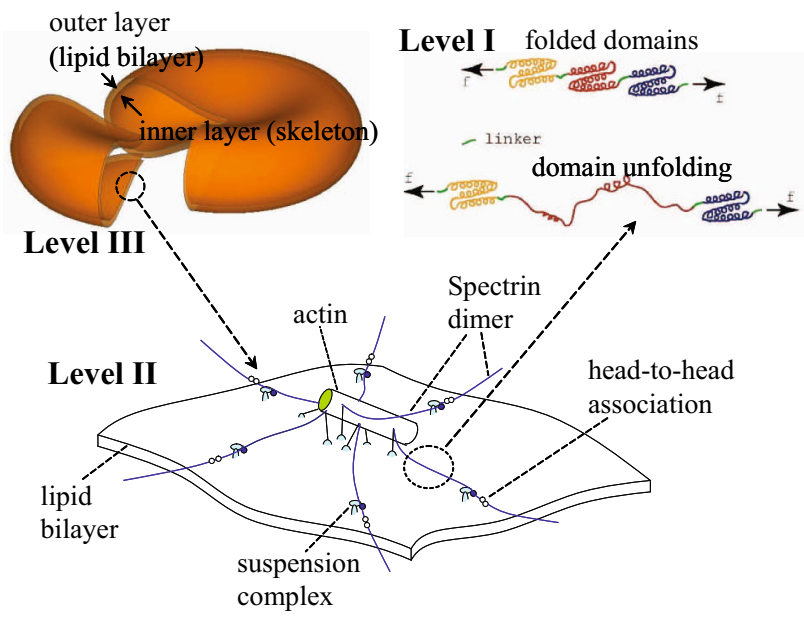

Fig. 2 Two-component RBC model using three-level hierarchical multiscale continuum method [21,22]

At Level I, Zhu and Asaro developed a model of spectrin using Monte-Carlo simulation [29]. Spectrin is the major RBC cytoskeleton protein that forms a triangular network. Rief et al. applied AFM to stretch a single spectrin and found that it can be modeled accurately using a nonlinear constitutive model called worm-like chain (WLC) $[23,24,43]$. Besides that, it was found that if the spectrin is stretched beyond its contour length, multiple protein domains may unfold to increase the contour length. In order to capture the full mechanical behavior of the spectrin, Zhu and Asaro took into account its domain unfolding feature by employing a Monte-Carlo method, as the probability of the unfolding is a function of the loading [29]. By simulating the unfolding of the spectrin, Zhu and Asaro got consistent force-displacement curves as in the AFM experiment [43]. In addition, the effect of stretching rate is also quantified. After these force-displacement curves are obtained, the information is passed as a database to the next level model (Level II).

At Level II, Zhu et al. developed a 3D JC model with molecular details using Brownian dynamics (BD), which is a basic protein complex unit in the RBC cytoskeleton [28]. In the JC, an actin protofilament is associated with four to six spectrins. Zhu et al. built an exact geometry model based on the state-of-the-art understanding of the molecular structure of a JC, including the binding sites between spectrins and actins [44]. They simulated thermal fluctuations of the RBC membrane using this model [28] and predicted the area and shear moduli of the cytoskeleton, as functions of membrane deformation $[21,28]$, which can be passed to the next cell level model (Level III).

At Level III, Peng et al. developed a whole cell model of RBCs using FEM and considered the normal and tangential interactions between the lipid bilayer and the cytoskeleton $[21,22]$. The cytoskeleton may slide against the lipid 
bilayer $[45,46]$, since the cytoskeleton is connected to the lipid bilayer through transmembrane proteins such as band 3 and glycophorin $\mathrm{C}$, which can move freely within the lipid bilayer. In order to describe the bilayer-cytoskeletal interaction accurately, we modeled the RBC membrane as two distinct layers. We employed an inner layer to present the cytoskeleton with finite area and shear stiffnesses, and negligible bending stiffness, and an outer layer to represent the lipid bilayer with zero shear stiffness, huge area stiffness, and significant bending stiffness. Vertical elastic interaction and tangential viscous friction between the lipid bilayer and the cytoskeleton are simulated using a contact algorithm based on the penalty method [47]. The bilayer-cytoskeletal friction coefficient is estimated based on the Stokes-Einstein relation and experimentally measured diffusivities of band 3 and glycophorin C in the lipid bilayer [48-50]. Reduced integration shell elements based on Mindlin theory were used to simulate both the outer and the inner layers [21].

Besides the elasticity, we also considered the viscosities of the lipid bilayer and the cytoskeleton, which play significant roles in dynamics processes such as tank treading. For example, it has been demonstrated that without considering the membrane viscosity, the predicted tank-treading frequency cannot match the experimental measurement [22,51]. Different from the existing study [51], we consider the viscosities of the lipid bilayer and the cytoskeleton separately using a generalized Voigt-Kelvin stress-strain relation [14]. Furthermore, we modeled the sliding between the bilayer and the skeleton as a viscous friction force. The viscous fluids surrounding the cell are incorporated in the following boundary element model of Stokes flow.

We applied a BEM to simulate the Stokes flows inside and outside of the RBC and coupled it with FEM to study the fluid-structure interaction. We used a staggered algorithm with explicit time integration [22]. For the interface dynamics in the Stokes flow with zero Reynolds number, we applied the boundary integral equation [9] so that the velocity $\mathbf{v}^{f}$ is given as

$$
\begin{aligned}
\mathbf{v}^{f}\left(\mathbf{x}_{0}\right)= & \frac{2}{1+\Lambda} \overline{\mathbf{v}}^{f}\left(\mathbf{x}_{0}\right) \\
& -\frac{1}{4 \pi \eta_{1}(\Lambda+1)} \iint_{\Gamma^{f b}} \mathbf{G}\left(\mathbf{x}, \mathbf{x}_{0}\right) \cdot \Delta \mathbf{t}^{f}(\mathbf{x}) \mathrm{d} \Gamma(\mathbf{x}) \\
& +\frac{1-\Lambda}{4 \pi(1+\Lambda)} \iint_{\Gamma^{f b}} \mathbf{v}^{f}(\mathbf{x}) \cdot \mathbf{T}\left(\mathbf{x}, \mathbf{x}_{0}\right) \cdot \mathbf{n}(\mathbf{x}) \mathrm{d} \Gamma(\mathbf{x}),
\end{aligned}
$$

where $\Lambda$ is the viscosity ratio, $\Gamma^{f b}$ is the membrane surface and $\overline{\mathbf{v}} f$ is the prescribed undisturbed velocity field of the shear flow. The vector $\Delta \mathbf{t}^{f}=\mathbf{t}^{f, 1}-\mathbf{t}^{f, 2}$ is the discontinuity in the interfacial surface traction, where $\mathbf{t}^{f, 1}$ is the traction in the outside surface $\Gamma^{f b, 1}$ of the interface, and $\mathbf{t}^{f, 2}$ is the traction in the inside surface $\Gamma^{f b, 2}$ of the interface. The surface traction is related to the nodal force through the principle of virtual work [52]. $f \int$ denotes the principal value integration.

$\mathbf{G}$ is the Green's function for velocity. Its components are

$G_{i j}\left(\mathbf{x}, \mathbf{x}_{0}\right)=\frac{\delta_{i j}}{\left|\mathbf{x}-\mathbf{x}_{0}\right|}+\frac{\left(x_{i}-x_{0_{i}}\right)\left(x_{j}-x_{0_{j}}\right)}{\left|\mathbf{x}-\mathbf{x}_{0}\right|^{3}}$,

where $\delta_{i j}$ is Kronecker's delta. T is the Green's function for stress. It can be written as

$T_{i j k}\left(\mathbf{x}, \mathbf{x}_{0}\right)=-6 \frac{\left(x_{i}-x_{0_{i}}\right)\left(x_{j}-x_{0_{j}}\right)\left(x_{k}-x_{0_{k}}\right)}{\left|\mathbf{x}-\mathbf{x}_{0}\right|^{5}}$.

Extensive validations have been conducted in each level to verify the models, such as optical tweezers stretching [21], micropipette aspiration [21], tank treading, and tumbling of RBCs in shear flow [22]. More importantly, the effect of stress-free cytoskeleton state on the tank-treading behaviors has been compared with the recent experiment in detail $[26,53,54]$. Besides validations, we predicted correlation between the occurrence of Sp unfolding and increase in the mechanical load upon individual skeleton-bilayer pinning points and related it to the vesiculation process [55]. The simulation results also show that during tank treading, the protein density variation is insignificant for healthy RBCs, but significant for cells with a smaller bilayer-cytoskeletal friction coefficient, which may be the case in hereditary spherocytosis [17]. We also predicted two different modes of motions for RBCs in shear flow [35] and studied the effect of stress-free state on the tank-treading motion [26,53,54]. We showed that the cell maintains its biconcave shape during tank-treading motions under low shear rate flows, by employing a spheroidal stress-free state in the cytoskeleton [26]. Furthermore, we numerically confirmed the hypothesis that, as the stress-free state approaches a sphere, the threshold shear rates corresponding to the establishment of tank treading decrease. By comparing with the experimental measurements [53], our study suggests that the stress-free state of RBCs is a spheroid that is close to a sphere, rather than the biconcave shape applied in existing models [26]. In addition, we also quantified the stability phase diagram of different motion modes in high shear rate flows and explored the effect of stress-free state on the phase diagram [54].

\subsection{Two-component model of RBC membranes using dissipative particle dynamics}

Besides the three-level hierarchical multiscale model of RBCs, Peng et al. also developed a DPD-based twocomponent model of RBC membranes by modeling the lipid bilayer and the cytoskeleton separately using two twodimensional triangulated networks with $N_{v}$ DPD particles [19]. We use DPD particles to model the fluids, which interact with the DPD network representing the lipid bilayer. We 
applied the bounce-back condition to enforce the non-slip condition between the bilayer and the fluids. In the following, we will describe DPD method for simulating the fluids first and then the details of the two-component RBC membrane model.

\subsubsection{Dissipative particle dynamics}

DPD is a mesoscopic numerical technique, which was originally developed to simulate fluids and polymers [56-58]. In the DPD simulations, fluids and polymers are represented by $N$ DPD particles, which interact with each other via pairwise potentials and their motions follow Newton's second law. Different from classical molecular dynamics, a DPD particle represents a cluster of atoms. Three kinds of pairwise interaction forces are applied between DPD particles $i$ and $j$ at positions $r_{i}$ and $r_{j}$, including conservative, dissipative, and random forces. They are written as

$F_{i j}^{C}=a_{i j} \omega\left(r_{i j}\right) \mathbf{e}_{i j}$,

$F_{i j}^{D}=-\gamma \omega^{2}\left(r_{i j}\right)\left(\mathbf{e}_{i j} \cdot \mathbf{v}_{i j}\right) \mathbf{e}_{i j}$,

$F_{i j}^{R}=\sigma \omega\left(r_{i j}\right) \zeta_{i j} \Delta t^{-1 / 2} \mathbf{e}_{i j}$,

where $\mathbf{r}_{i j}=\mathbf{r}_{i}-\mathbf{r}_{j}, r_{i j}=\left|\mathbf{r}_{i j}\right|, \mathbf{e}_{i j}=\mathbf{r}_{i j} / r_{i j}$, and $\mathbf{v}_{i j}=\mathbf{v}_{i}-\mathbf{v}_{j}$. The strengths of conservative, dissipative, and random forces are specified by constants $a_{i j}, \gamma$, and $\sigma$, respectively. $\Delta t$ is the time step size, and $\zeta_{i j}$ is a random number with zero mean and unit variance. $\omega\left(r_{i j}\right)$ is a weight function given by

$\omega\left(r_{i j}\right)= \begin{cases}1-r_{i j} / r_{c} & r_{i j}<r_{c} \\ 0 & r_{i j} \geq r_{c}\end{cases}$

where the cutoff radius is defined by $r_{c}$. Due to the dissipation-fluctuation theorem, the coefficients of random and dissipative forces follow a relationship $\sigma^{2}=2 \gamma k_{B} T$, where $k_{B}$ is the Boltzmann constant and $T$ is the temperature, so that the two forces act a thermostat. Furthermore, due to the soft core interaction in the conservative force, we are able to use much larger time steps than that in the MD simulations. In summary, DPD is efficient simulation technique for modeling fluids and polymers.

\subsubsection{Membrane viscoelasticity and bilayer-cytoskeletal interactions of the two-component RBC model}

The RBC membrane is modeled by two different components in the two-component DPD model, i.e., the lipid bilayer and the cytoskeleton, as shown in Fig. 3. Each component is represented by a $2 \mathrm{D}$ triangular network with $N_{v}$ DPD particles.

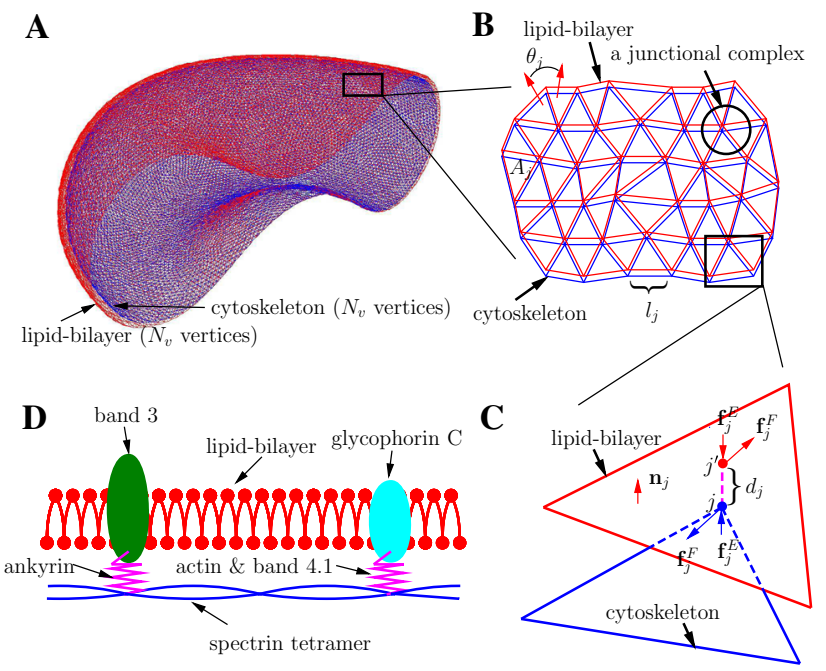

Fig. 3 A Two-component DPD model of a RBC. B Local triangular networks of the two-component model. C Normal and tangential interactions between the lipid bilayer and the cytoskeleton. D Physical picture of the local bilayer-cytoskeletal interaction [19]

The total elastic energy of the RBC membrane is written as

$U=U_{s}+U_{b}+U_{a+v}+U_{\mathrm{int}}$,

where $U_{s}$ is the cytoskeleton elastic energy, given by the WLC potential and a repulsive power potential as

$U_{s}=\sum_{j \in 1 \ldots N_{s}}\left[\frac{k_{\mathrm{B}} T l_{m}\left(3 x_{j}^{2}-2 x_{j}^{3}\right)}{4 p\left(1-x_{j}\right)}+\frac{k_{p}}{(n-1) l_{j}^{n-1}}\right]$,

where $l_{j}$ and $l_{m}$ are the spectrin natural length and contour length, $N_{s}$ is the number of spectrins, while $x_{j}=l_{j} / l_{m} . p$ is the persistence length, $k_{\mathrm{B}}$ is the Boltzmann constant, $T$ is the temperature, and $k_{p}$ is the power term constant.

$U_{b}$ is the elastic energy of the lipid bilayer, which is written as

$U_{b}=\sum_{j \in 1 \ldots N_{s}} k_{b}\left[1-\cos \left(\theta_{j}-\theta_{0}\right)\right]$,

where $k_{b}$ is the bending coefficient and $k_{b}=2 k_{c} / \sqrt{3}$, where $k_{c}$ is the bending stiffness of the bilayer. $\theta_{0}$ is the spontaneous angle and $\theta_{j}$ is the instantaneous angle between two adjacent triangles as shown in Fig. 3B. Furthermore, $U_{a+v}$ is the energy due to cell area and volume conservation, which is written as

$U_{a+v}=\sum_{j \in 1 \ldots N_{t}} \frac{k_{l}\left(A_{j}-A_{0}\right)^{2}}{2 A_{0}}+\frac{k_{v}\left(V^{\mathrm{tot}}-V_{0}^{\mathrm{tot}}\right)^{2}}{2 V_{0}^{\mathrm{tot}}}$,

where $N_{t}$ is triangle number, $A_{0}$ is the initial triangle area and $A_{j}$ is the current triangle area as shown in Fig. 3B. $V_{0}^{\text {tot }}$ 
is the initial volume of a RBC and $V^{\text {tot }}$ is the current volume. $k_{l}$ and $k_{v}$ are penalty constants.

In addition to the membrane elasticity, we also consider the viscosities of the lipid bilayer and the cytoskeleton by adding dissipative and random forces as

$$
\begin{aligned}
\mathbf{F}_{i j}^{D, k}= & -\gamma_{k}^{T} \mathbf{v}_{i j}-\gamma_{k}^{C}\left(\mathbf{v}_{i j} \cdot \mathbf{e}_{i j}\right) \mathbf{e}_{i j}, \\
\mathbf{F}_{i j}^{R, k} \Delta t= & \sqrt{2 k_{\mathrm{B}} T}\left(\sqrt{2 \gamma_{k}^{T}} d \overline{\mathbf{W}_{i j}^{S}}\right. \\
& \left.+\sqrt{3 \gamma_{k}^{C}-\gamma_{k}^{T}} \frac{\operatorname{tr}\left[d \mathbf{W}_{i j}\right]}{3} \mathbf{1}\right) \cdot \mathbf{e}_{i j},
\end{aligned}
$$

where $k=b, s$ stands for the lipid bilayer or the cytoskeleton, respectively. $\gamma_{k}^{T}$ and $\gamma_{k}^{C}$ are dissipative coefficients with a condition $3 \gamma_{k}^{C^{k}}>\gamma_{k}^{T} ; \mathbf{e}_{i j}$ and $\mathbf{v}_{i j}$ are the relative position and velocity. $d \overline{\mathbf{W}_{i j}^{S}}=d \mathbf{W}_{i j}^{S}-\operatorname{tr}\left[d \mathbf{W}_{i j}^{S}\right] \mathbf{1} / 3$, where $d \mathbf{W}_{i j}$ is the Wiener increment. The Wiener increment $d \mathbf{W}_{i j}(t)=$ $\mathbf{W}_{i j}(t+\Delta t)-\mathbf{W}_{i j}(t)$ over a time step $\Delta t$ is a random variable drawn from a normal distribution with zero mean and a timestep variance $\mathcal{N}[0, \Delta t]$. The membrane viscosities can be obtained as

$\eta_{k}=\sqrt{3} \gamma_{k}^{T}+\frac{\sqrt{3} \gamma_{k}^{C}}{4}$

Viscosity values are taken from the literature [59].

To describe the bilayer-cytoskeletal interaction, we add another term $U_{\text {int }}$ as

$U_{\mathrm{int}}=\sum_{j \in 1 \ldots N_{b s}} \frac{k_{b s}\left(d_{j}-d_{j 0}\right)^{2}}{2}$

where $k_{b s}$ represents the elastic stiffness of the bonds between the bilayer and the cytoskeleton, and $N_{b s}$ is the number of vertical bonds, including the bonds between the transmembrane proteins (band-3 and glycophorin C) and spectrins. We simulate the bilayer-cytoskeletal interaction by a tangential friction force and a normal viscoelastic force as shown in Fig. $3 \mathrm{C}, \mathrm{D} . d_{j}$ is current bond length; $d_{j 0}$ is the initial bond length. Experiments show that $d_{j 0} \approx 30 \mathrm{~nm}$ [60]. We employed a master-slave penalty contact algorithm to calculate the bilayer-cytoskeletal interaction force [47]. The elastic interaction force on a cytoskeletal vertex is given as

$\mathbf{f}_{j}^{E}=k_{b s}\left(d_{j}-d_{j 0}\right) \mathbf{n}_{j}$,

where $\mathbf{n}_{j}$ is the normal unit vector. We also add a vertical damping force as

$\mathbf{f}_{j}^{D}=-c_{b s}\left(\mathbf{v}_{j} \cdot \mathbf{n}_{j}\right) \mathbf{n}_{j}$,

where $\mathbf{v}_{j}$ is the relative velocity and $c_{b s}$ is the damping coefficient. The bilayer-cytoskeletal viscous friction force is given as
$\mathbf{f}_{j}^{F}=-f_{b s}\left[\mathbf{v}_{j}-\left(\mathbf{v}_{j} \cdot \mathbf{n}_{j}\right) \mathbf{n}_{j}\right]$,

where $f_{b s}$ is the friction coefficient.

To keep the constant temperature, we add another random force [61] as

$\mathbf{f}_{j}^{R} \Delta t=\sqrt{2 k_{\mathrm{B}} T}\left(\sqrt{2 f_{b s}} d \mathbf{W}_{i j}^{A}+\sqrt{3 c_{b s}} \frac{\operatorname{tr}\left[d \mathbf{W}_{i j}\right]}{3} \mathbf{1}\right) \cdot \mathbf{n}_{j}$,

where $d \mathbf{W}_{i j}^{A}=\left(d \mathbf{W}_{i j}-d \mathbf{W}_{j i}\right) / 2$ is the anti-symmetric part of the Wiener increment.

To summarize, the total bilayer-cytoskeletal interaction force is given as

$\mathbf{f}_{j}^{\mathrm{int}}=\mathbf{f}_{j}^{E}+\mathbf{f}_{j}^{D}+\mathbf{f}_{j}^{F}+\mathbf{f}_{j}^{R}$.

The two-component RBC DPD model has been validated against more than six different experiments by using the same set of input parameters, such as micropipette aspiration, membrane fluctuations, tank-treading motion in shear flow, bilayer tethering in channel flow, passage through microfluidic channel, and twisting torque cytometry. More importantly, based on this two-component DPD model, several controversies and issues in RBC mechanics have been resolved. The micropipette aspiration experiments of measuring both the area moduli of the cytoskeleton $\left(k_{s}\right)$ and the lipid bilayer $\left(k_{l}\right)$ were simulated accurately using the same set of input parameters [62-64], although these two area moduli differ by five orders of magnitude. It was found that due to the explicit incorporation of bilayer-cytoskeletal elastic interaction, the two-component model matches the thermal fluctuations experiments better than the one-component model [65]. It was also found that the dependence of the tank-treading frequency on the shear rate follows a linear relationship for a narrow channel but a nonlinear one for a wide channel, which resolves a controversy in the literature [66]. It has been demonstrated that the tank-treading motion is too fast for the bilayer-cytoskeletal slip to occur for healthy RBCs $[45,46]$. The bilayer-cytoskeletal interaction strength was predicted by simulating the RBC tethering in the channel flow experiment [67]. In addition, using this two-component model, Li et al. also studied RBC deformability in a small microfluidic channel and investigated the membrane mechanical properties [68]. The twisting torque cytometry was simulated and it was found that the predicted rheological properties of the RBC membrane match well with experimental measurements. In these experiments, $\mathrm{Li}$ et al. also showed that bilayer-cytoskeletal interactions are important for cell membrane mechanical, rheological, and dynamical properties [68]. 


\subsection{Two-component RBC model using coarse-grained molecular dynamics}

In a shorter length scale, Li and Lykotrafitis developed a twocomponent coarse-grained MD model for RBC membranes by adopting the one-particle-thick solvent-free model [75] for the lipid bilayer and a WLC network [30] or detailed free-jointed chain model [31] for the cytoskeleton. Zhang et al. also applied a similar coarse-grained model to study the stiffening effects of nanoscale knobs on human RBCs infected with Plasmodium falciparum malaria parasite [69].

In the one-particle-thick solvent-free model of the lipid bilayer, the following interaction potential function was adopted to enforce the DPD particles in a monolayer to form a one-particle-thick layer without losing particles,

$$
\begin{aligned}
u\left(\mathbf{n}_{i}, \mathbf{n}_{j}, \mathbf{x}_{i j}\right)= & u_{R}\left(r_{i j}\right)+u_{A}\left(r_{i j}\right) \\
& +\alpha\left[a\left(\mathbf{n}_{i}, \mathbf{n}_{j}, \mathbf{x}_{i j}\right)-1\right] u_{A}\left(r_{i j}\right)
\end{aligned}
$$

where $r_{i j} \equiv\left|\mathbf{x}_{i j}\right|, \mathbf{x}_{i j}$ is the relative position vector between two particles, and $\mathbf{n}_{i}$ is the unit directional vector of each particle. The attractive and repulsive potentials $u_{A}\left(r_{i j}\right)$ and $u_{R}\left(r_{i j}\right)$ are given by

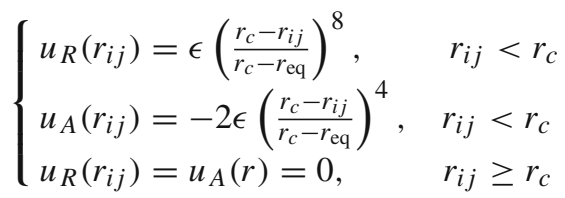

where the coefficient $\alpha=1.55$ is specified to maintain the correct bending stiffness, $\epsilon=4.54 k_{B} T$ is the depth of the energy well, $r_{c}=2.6 d$ is the cut off length, $r_{\mathrm{eq}}=2^{1 / 6} d$ is the equilibrium length, and $d$ is the unit of length.

$$
\begin{aligned}
a= & \left(\mathbf{n}_{i} \times \mathbf{x}_{i j}\right) \cdot\left(\mathbf{n}_{j} \times \mathbf{x}_{i j}\right) \\
& +\sin \theta_{0}\left(\mathbf{n}_{j}-\mathbf{n}_{i}\right) \cdot \mathbf{x}_{i j}-\sin ^{2} \theta_{0} .
\end{aligned}
$$

$\theta_{0}$ is the spontaneous angle associated with the bilayer spontaneous curvature.

The translational and rotational motions are described by

$$
\begin{aligned}
& m_{i} \ddot{\mathbf{x}}_{i}=-\frac{\partial V_{i}}{\partial \mathbf{x}_{i}} \\
& I_{i} \ddot{\mathbf{n}}_{i}=-\frac{\partial V_{i}}{\partial \mathbf{n}_{i}}+\left(\frac{\partial V_{i}}{\partial \mathbf{n}_{i}} \cdot \mathbf{n}_{i}\right) \mathbf{n}_{i}-I_{i}\left(\dot{\mathbf{n}}_{i} \cdot \dot{\mathbf{n}}_{i}\right) \mathbf{n}_{i}
\end{aligned}
$$

where $V_{i}=\sum_{j=1}^{N} u\left(\mathbf{x}_{i j}, \mathbf{n}_{i}, \mathbf{n}_{j}\right) . I_{i}$ and $m_{i}$ are the inertial moment and mass of a particle, and $\mathbf{x}_{i}$ is the position vector of a particle. Li and Lykotrafitis applied similar interaction potentials between the lipid particle and band 3 particle.

To incorporate the cytoskeleton of spectrin, $\mathrm{Li}$ and Lykotrafitis first applied WLC model to simulate the spectrin between the band 3 and actin in the one-particle-thick model [30], each of which are represented by a CG particle. In a refined model, $\mathrm{Li}$ and Lykotrafitis employed 39 particles connected by unbreakable springs to represent the spectrin instead of a single WLC chain and also considered the interaction between the spectrins and the lipids by LJ potentials [31], as shown in Fig. 4.

The shear modulus predicted by their model is consistent with the experimental measurement [30]. The model has also been validated by comparing the results with experimentally measured viscosity and thermal fluctuations of the RBC membrane [31]. Li and Lykotrafitis found that the RBC membrane bending stiffness and the transmembrane protein diffusivity can be accurately captured by adjusting the interaction potential parameters [30]. They found that at higher strain rates the shear stress is mainly determined by the viscosity of the lipid bilayer, and at lower shear strain rates the shear stress is mainly due to the cytoskeleton. Using the extended model [31], they discovered that the pressure imposed by the cytoskeleton on the lipid bilayer is reduced due to the defects of the connections between the cytoskeleton and the lipid bilayer, which might occur in spherocytes. More importantly, by comparison, they showed that this pressure is even more significantly reduced if there are defects in the dimer-dimer association of a spectrin filament, which might happen in elliptocytes [17]. They also explored the attractive forces between the

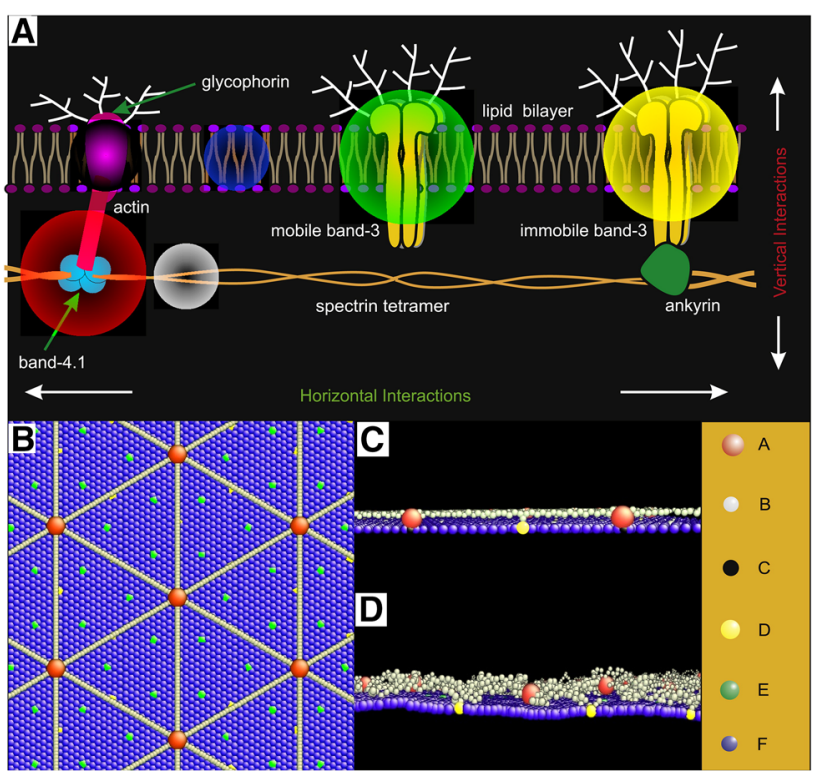

Fig. 4 Two-component RBC model using coarse-grained MD [30,31]. (A) The structure of $\mathrm{RBC}$ membranes. The spheres with different colors represent different components: junctional complex (red), lipid particle (blue), spectrin particle ( gray) glycophorin particle (black), fixed band3 (yellow), and mobile band-3 (green). (B) Undeformed membrane in topview. $(C)$ Undeformed membrane in side view. $(D)$ Deformed membrane in side view. (Copied from [31], Colour figure online) 
lipid bilayer and the spectrin filaments and showed that it leads to increased pressure from the cytoskeleton to the lipid bilayer.

\subsection{Comparison of different $\mathrm{RBC}$ models and related experiments}

The main features and applications of some existing mesoscale RBC models are summarized in Table 1. The main differences are the molecular details considered and the length scales these model can reach. The two-component CG model focused on the detailed bilayer-cytoskeletal interaction and transmembrane protein diffusion, but it is too expensive to employ it to study a whole cell with current methodology. The two-component DPD model studied the whole cell behavior and explore the bilayer-cytoskeletal in a simplified way. The three-level multiscale approach is developed to bridge three models in different length scale, but due to the information passing, some information is lost, such as thermal fluctuations in the whole cell level.

There are still plenty of room to improve these models. For example, only one-way coupling has been done for the threelevel multiscale RBC model, and two-way coupling can be added to study how the deformation in the macroscale can influence the dynamics of the proteins in the microscale. In addition, future effort will be put on how to improve the efficiency of the two-component CGMD RBC model in order to apply it in the whole cell scale and coupled it with fluid motion. Moreover, smoothed dissipative particle dynamics (SDPD) and smoothed particles hydrodynamics (SPH) have been also applied to study RBCs models [76-78]. Compared to conventional DPD, SDPD adopts advantages from smoothed particles hydrodynamics (SPH) [79], such as thermal consistency and specification of viscosity.
Since the RBC is a model system for cell mechanics and biological membranes, RBC models can be applied in other systems as well. For example, recently we extended the twocomponent RBC DPD model to study the blood vessel walls by considering the isotropic matrix and the anisotropic collagen fibers separately [80]. Furthermore, we can also apply RBC models to study the lipid bilayer and the actin cortex network or the intermediate filament network in general eukaryotic cells, because some of them share similar structures as the spectrin network of RBCs, such as the auditory outer hair cells and the nucleus lamina [33,81].

\section{Mesoscale modeling of DNA dynamics}

Mesoscale modeling of DNA has granted insights into DNA mechanics, conformation, and dynamics in free solution [8294] and for DNA in nanoslits [95-111] and nanochannels [112-124]. In particular, there has been significant interest in the validation of polymer theory using DNA as a model polymer due to ease of direct observation of single DNA molecules and its highly monodisperse nature [104,125144], with potential applications in genome analysis and sequencing [145-150]. Here, we will focus on a challenging problem for mesoscale coarse-grained DNA models : DNA translocation through a nanopore with applications for DNA separation and sequencing.

It is challenging to accurately model DNA molecules in solution due to the need to simultaneously resolve long-range interactions (i.e., hydrodynamic and electrostatic) and shortrange interactions (i.e., local rigidity, hydrophobic, entropic). DNA properties of interest can span several orders of magnitudes in terms of length and time as shown in Fig. 5. The computational cost of calculating the continuum hydrody-
Table 1 Comparison of RBC models

\begin{tabular}{|c|c|c|}
\hline Models & Main characteristics & Applications \\
\hline $\begin{array}{l}\text { Three-level } \\
\text { multiscale }[21,22]\end{array}$ & $\begin{array}{l}\text { FEM, } L D \text {, and MC, spanning from } \\
\text { protein to cell }\end{array}$ & $\begin{array}{l}\text { Micropipette vesiculation, dynamics of } \\
\text { JCs, spectrin unfolding }\end{array}$ \\
\hline $\begin{array}{l}\text { One-component } \\
\text { DPD }[25,51]\end{array}$ & $\begin{array}{l}\text { Whole cell, coupled with fluids, } \\
\text { systematic coarse-graining }\end{array}$ & $\begin{array}{l}\text { Thermal fluctuations, tank treading, } \\
\text { microfluidics }\end{array}$ \\
\hline $\begin{array}{l}\text { Two-component } \\
\text { DPD }[19,68]\end{array}$ & $\begin{array}{l}\text { Bilayer-cytoskeletal tangential and } \\
\text { vertical interaction in molecular } \\
\text { level, whole cell }\end{array}$ & $\begin{array}{l}\text { Bilayer-cytoskeletal slip, bilayer } \\
\text { tethering, thermal fluctuations }\end{array}$ \\
\hline $\begin{array}{l}\text { Two-component CG } \\
{[30,31,69]}\end{array}$ & $\begin{array}{l}\text { One-particle-thick bilayer model, } \\
\text { Small piece of membrane }\end{array}$ & $\begin{array}{l}\text { Transmembrane protein diffusion, effects } \\
\text { of protein deficiencies, budding of } \\
\text { bilayer }\end{array}$ \\
\hline $\begin{array}{l}\text { Two-component } \\
\text { MCPD [27] }\end{array}$ & Coarse-graining, whole cell & Tube flow, tank treading \\
\hline LBM $[70,71]$ & $\begin{array}{l}\text { Fluid-structure interaction, whole } \\
\text { cell }\end{array}$ & Blood rheology, margination \\
\hline $\begin{array}{l}\text { Continuum } \\
{[20,37,72-74]}\end{array}$ & $\begin{array}{l}\text { Uniform property, no molecular } \\
\text { details }\end{array}$ & Tank treading, optical tweezers stretching \\
\hline
\end{tabular}


Fig. 5 The characteristic length scales of a DNA molecule range from angstrom to millimeters

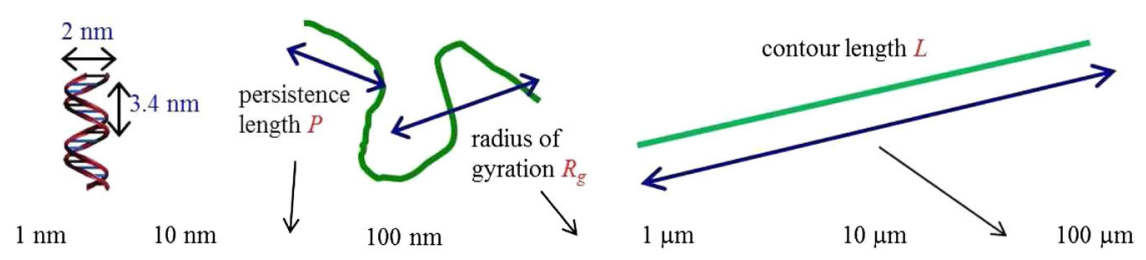

Nanochannels

Microchannels

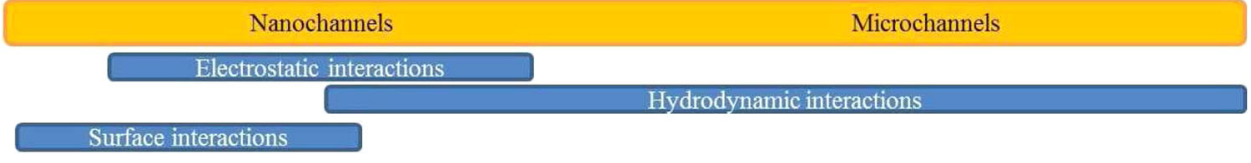

namic field by solving the non-linear Navier-Stokes equation is typically of order $\mathrm{O}\left(N^{2.25}\right)$, where $N$ is the number of mesoscale beads in the system, rendering computation of large systems prohibitively expensive. Recently, fully coupled mesoscale methods such as lattice Boltzmann (LB) [151,152], fast-multipole integration [153, 154], and particlebased methods such as dissipative particle dynamics (DPD) $[155,156]$, and multi-particle collision dynamics (MPCD) [85,157] have demonstrated $\mathrm{O}(N \log (N))$ efficiency for capturing long-range interactions such as hydrodynamics interaction. These developments have spurred new interests in the investigation of DNA dynamics in microfluidic flow and other systems.

\subsection{Coarse-grained DNA models}

Mesoscale models of DNA dynamics in free solution often neglect DNA stiffness and sequence specificity, which are very important for understanding DNA properties in nanoconfinement. There are two major bottlenecks for modeling DNA dynamics in nanoscale confinement: (1) the large number of degrees of freedom needed to capture short-range interactions and (2) the high cost to accurately calculate the long-range electrostatic and hydrodynamic forces. Novel multiscale methods that will allow efficient computation of long-range (hydrodynamic and electrostatic) and short-range (van der Waals) forces are needed to understand DNA dynamics in nanochannels and nanopores.

Efficient computation of DNA properties in different systems requires choosing a multiscale DNA model by a careful examination of the important physical interactions, as shown in Fig. 6. To model the properties of large DNA molecules in free solution, a flexible bead-spring polymer model coupled with a continuum fluid model may be used to accurately capture long-time DNA dynamics [91,158,159]. Each spring represents a large number of Kuhn segments. For small spring extension in the linear force-extension regime such as DNA equilibrium properties, the harmonic or finitely extensible non-linear elastic spring potential could accurately capture the extensional force $[160,161]$. However, for large spring extension such as DNA under strong shear or extensional

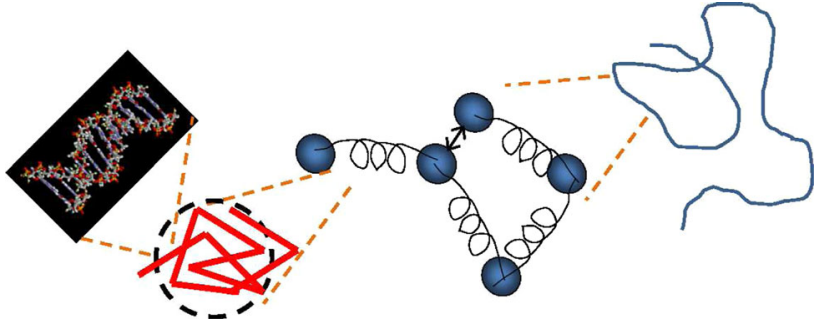

Fig. 6 Different scales of DNA model from a continuous string to atomistic

deformation, the WLC model is needed to accurately capture the DNA dynamics $[82,162]$.

In principle, a fully flexible chain could be used to model DNA properties of millimeter length scales. However, this inherently neglects intermolecular physics for length scales of order Kuhn length $(\approx 100 \mathrm{~nm})$ and smaller due to DNA backbone stiffness. For DNA in nanochannels, DNA semi-flexibility due to the double-helix structure must be accounted for. A coarse-grained model that includes inter-segment bending potential to correspond to the DNA persistence length may capture DNA rigidity and the forceextension relation [106]. Recent studies have shown that the semi-flexible chain model captures DNA dynamics in nanoslits and nanochannels [101-103,107-109,119,121123]. However, the spring length of the semi-flexible chain is typically of length $2-10 \mathrm{~nm}$, and the model is most useful for modeling shorter DNA molecules with contour length of order $10 \mu \mathrm{m}$ due to the computational cost. In order to study DNA dynamics in nanopores, the model must be able to capture nucleotide-pore and base-base interactions. Models such as the 3-site-per-nucleotide DNA model $[86,163,164]$ have been shown to capture DNA hybridization kinetics and also DNA structural rigidity. It may be a promising start point to accurately model sequence-dependent DNA translocation through a nanopore.

\subsection{Mesoscale modeling of DNA/polymer translocation through nanopores}

Nanopore translocation has been extensively studied due to its promising application for rapid, label-free, and real-time 
Table 2 Comparison of DNA models

\begin{tabular}{|c|c|c|}
\hline Models & Main characteristics & Applications \\
\hline All-atom MD [176,177] & $\begin{array}{l}\text { Capturing atomic details of up to } 10 \mathrm{~nm} \text { ds-DNA } \\
\text { segments }\end{array}$ & Current signature of different base pairs \\
\hline $3 \mathrm{PN}[86,163,164]$ & $\begin{array}{l}\text { Differentiate coarse-grained nucleic acids of up to } \\
100 \mathrm{~nm} \text { ds-DNA segments }\end{array}$ & $\begin{array}{l}\text { Studies of the dynamics of DNA hybridization and } \\
\text { structure, properties of single base-pair mismatch, } \\
\text { DNA-protein interactions }\end{array}$ \\
\hline $\begin{array}{l}\text { CG semi-flexible polymer } \\
{[106,225,226]}\end{array}$ & $\begin{array}{l}\text { Capture the physics of flow-polymer interaction on } \\
\text { length scales } 5 \mathrm{~nm} \text { or greater without single nucleic } \\
\text { acid resolution }\end{array}$ & $\begin{array}{l}\text { Studies of DNA dynamics and conformation in } \\
\text { micro- and nanochannels, DNA-protein } \\
\text { interactions }\end{array}$ \\
\hline $\begin{array}{l}\text { CG flexible polymer } \\
{[83,88,159,180]}\end{array}$ & $\begin{array}{l}\text { Capture the physics of flow-polymer interaction on } \\
\text { length scales } 100 \mathrm{~nm} \text { or greater without single nucleic } \\
\text { acid resolution }\end{array}$ & $\begin{array}{l}\text { Studies of DNA dynamics and conformation in } \\
\text { microchannels }\end{array}$ \\
\hline DPD [156,187,227] & $\begin{array}{l}\text { Coarse-grained polymer model accounting for } \\
\text { hydrodynamic interactions }\end{array}$ & DNA dynamics in micro-flow \\
\hline LB [152,202-205] & $\begin{array}{l}\text { Coarse-grained polymer model accounting for } \\
\text { hydrodynamic interactions }\end{array}$ & DNA dynamics in flow \\
\hline Continuum/FEM [111] & $\begin{array}{l}\text { Coarse-grained polymer model, flow-polymer } \\
\text { interaction }\end{array}$ & DNA dynamics in flow \\
\hline
\end{tabular}

DNA sequencing [165-171]. The length scale of interest covers that of a single nucleotide with size of $s \sim 0.34 \mathrm{~nm}$ to DNA persistence length $(\sim 50 \mathrm{~nm})$ to that of the DNA contour length with $N$ bases ( $N s$ can be more than several $\mu \mathrm{m})$, as illustrated in Fig. 5. Similarly, the time scale ranges from that of the nucleotide diffusion time $\left(\sim s^{2} f_{c} / k_{B} T\right.$, where $f_{c}$ is the friction coefficient) to the translocation time ( $\sim$ contour length/translocation velocity; translocation velocity $\sim$ electrophoretic force/friction coefficient). Up to six orders of magnitudes of variation in characteristic length and time need to be captured, which is a severe challenge for current computational capabilities. In order to model DNA translocation through a nanopore, DNA interactions with the nanopore itself must be accurately included, and thus the smallest characteristic length scale must be smaller than the nanopore size. The conformation of DNA segments vary from rod-like $(<50 \mathrm{~nm})$ to coil-like. Muthukumar first analyzed the translocation of a string-like polymer through a hole and found that the translocation time $\sim N^{2}$ without external force (thus diffusion dominated) and $\sim N$ for driven translocation [171]. More recent coarse-grained models that include hydrodynamic interactions and more detailed DNA structure have found slightly different power-law exponents [172-175]. Thus, different qualitative trends in the translocation dynamics are expected with the inclusion of more molecular details and hydrodynamic interactions.

Even so, all-atom modeling of even a short DNA fragments with only one persistence length $(\approx 50 \mathrm{~nm})$ in solution would still be prohibitively expensive, requiring more than $10^{8}$ atoms. Bhattacharya et al. applied all-atom MD to study DNA translocation through protein nanopore Mycobacteria smegmatic porin A (MspA). However, it takes days for using thousands of CPUs to simulate the translocation of ten nucleotides through a nanopore for $1 \mu \mathrm{s}$ [176]. Li et al. also applied MD to study the translocation of a singlestranded DNA through nanopores on graphene membranes [177]. Another challenge due to the cost of performing one set of MD simulations is that it is difficult to collect large samples for statistical evaluation. On the other hand, coarsegrained models have been employed extensively to study polymer translocation through a pore [174,175,178-183]. However, it remains challenging to accurately incorporate nucleotide characteristics, hydrodynamic interactions, electrostatic interactions, and DNA-pore interactions together in a complete model. In order to overcome these challenges, two recently developed techniques, dissipative particle dynamics (DPD) and lattice Boltzmann (LB), are discussed here and the main characteristics are summarized in Table 2 . The solvent modeling, DPD, LB, and continuum modeling are separately consistent with the chosen polymer model.

\subsubsection{Modeling of DNA/polymer translocation using dissipative particle dynamics}

In recent years, DPD has been applied to study polymer translocation through a nanopore. DPD was demonstrated to be efficient in modeling polymer translocation [183-185]. Kapahnke et al. first employed 3D DPD simulation to investigate the translocation of a polymer driven by a solvent quality contrast across different sides of a membrane [185]. Duong-Hong et al. applied DPD to study the combined DNA electrophoretic flow and electroosmotic flow (EOF) in nanofluidic devices and considered the EOF effect by utilizing a slip velocity as the wall boundary [186]. They also com- 
pared the DPD simulation results with the experiments [186]. In order to consider explicitly the electrostatic interactions between particles and avoid the singularity caused by the Coulomb attraction between particles, hard-core interactions have often been employed to prevent $[156,187]$. For example, in addition to the typical DPD interactions shown in Eqs. 4-6, Li et al. used hard-core Morse interaction and a screened Coulombic interaction in the following form [156]:

$$
\mathbf{F}_{i}=\sum_{i \neq j}\left(\mathbf{F}_{i j}^{C}+\mathbf{F}_{i j}^{D}+\mathbf{F}_{i j}^{R}+\mathbf{F}_{i j}^{M}+\mathbf{F}_{i j}^{S}+\mathbf{F}_{i j}^{\text {Coulomb }}\right)+\mathbf{F}_{i j}^{e x t},
$$

where $\mathbf{F}_{i j}^{C}, \mathbf{F}_{i j}^{D}$, and $\mathbf{F}_{i j}^{R}$ are the DPD forces shown in Eqs. 4-6. $\mathbf{F}_{i j}^{\text {Coulomb }}$ is the electrostatic interaction forcem, and $\mathbf{F}_{i j}^{M}$ is a repulsive force for preventing overlapping of ions and counterions. $\mathbf{F}_{i j}^{\text {ext }}$ is the external force field.

The bond interaction between DNA segments is given as

$\mathbf{F}_{i j}^{s}=k_{\text {bond }}\left(1-\frac{r_{i j}}{r_{0}}\right) \mathbf{n}_{i j}$,

where $k_{\text {bond }}$ is the strength of the harmonic bond and $\mathbf{n}_{i j}$ is the unit direction vector of the bond. $r_{i j}$ is the bond length, and $r_{0}$ is the initial bond length.

Based on the DPD approach, He et al. [184] found that the translocation time also scales with the force field strength as $\tau \sim \mathrm{E}^{-0.48 \pm 0.01}$. For unforced translocation, it was found that the translocation time follows a scaling $\tau \sim N^{\beta}$ with $\beta$ approximate to 2.24 [185]. Guo et al. observed single-file and double-folded translocation processes by studying the conformational changes of the polymer during the translocation [188] and showed that the average translocation time steadily decreases with the increase of fluid flux. It was also found that the chain rigidity plays an important role on the dynamics of polymer chain translocation [189]. Feng et al. applied DPD with two different dissipative radii in three dimensions and compared with the results of Langevin dynamics (LD) method [187]. Their results showed that the scaling relationship between the translocation time and the polymer chain length is $\tau \sim N^{0.94}$ by DPD method with small dissipative radius and $\tau \sim N^{0.98}$ with large dissipative radius. It was also found that the translocation time predicted by LD is larger than that by DPD method. Li et al. showed that the average translocation time $\tau$ scales with the polymer length $N$ as $\tau \sim N^{\beta}$ [190], where $\beta$ depends on the solvent quality [156] and could be adjusted by varying the repulsive parameter $a_{P S}$ between the polymer and the solvent in Eq. 4 as

$a_{P S}=a_{i j}(1+\epsilon)$,

where $\epsilon$ is a parameter that determines the solvent quality. Poor solvent quality can be modeled with $\epsilon>0$, and good solvent quality is modeled with $\epsilon<0$. A comparative study of DPD and modified LD was carried out for polymer translocation [191], and it determined that the inclusion of hydrodynamic interactions increases the translocation probability and decreases the translocation time. It was also found that the wall interfacial property plays an important role in the dynamics of polymer translocation. Uniform hydrostatic force and uniform electrostatic force have been applied to study the polymer translocation, and it was found that the scaling correlations $\tau \sim \mathrm{E}^{-\xi}$ and $\tau \sim N^{\beta}$ are valid only for coil-like chains [155]. The translocation process of star polymers through a nanochannel was investigated, and it was found that the translocation time shows a power-law dependence on the number of arms outside the channel and very weakly dependent on the number of arms in the channel [192].

\subsubsection{Simulation of electrostatic effect on DNA translocation using dissipative particle dynamics}

There are different ways to consider the electrostatic effect in DNA translocation in the DPD simulations. One way is to considered the electroosmotic flow effect by utilizing a slip velocity as the wall boundary [186,193]. But if the electrostatic interaction between particles are explicitly calculated, as shown in Fig. 7, there are two major challenges. The first challenging is that a soft core potential is usually used in the mesoscale modeling methods such as DPD, so that the ion and counterion may form a cluster pair due to the strong electrostatic interaction since there is no hard-core interaction to prevent overlap of ions and counterions $[194,195]$.

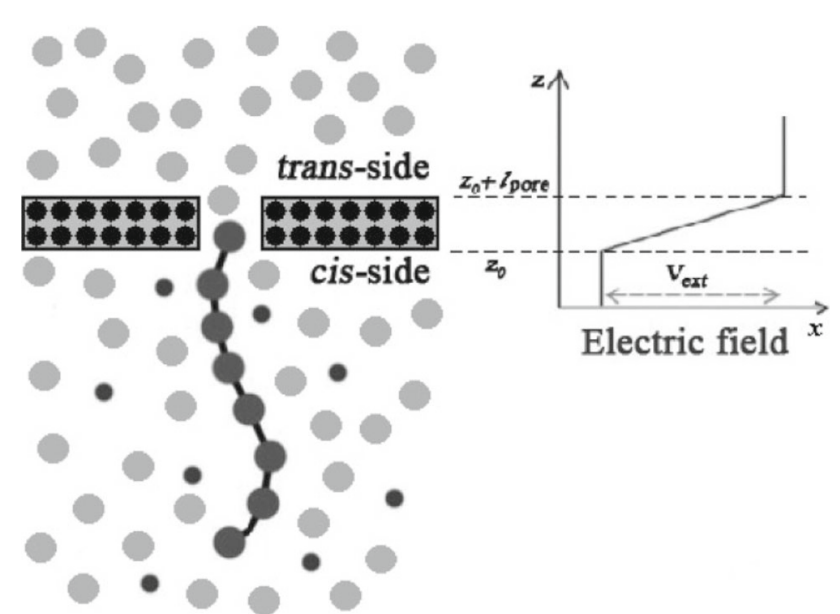

Fig. 7 Schematic representation of the DPD simulation model for a polymer chain and a narrow pore. In this figure, the neutral gray, light gray, and black color circles represent the polymer, solvent, and wall particles, respectively. In addition, the dark gray color circles represent the counterions. (Copied from [156]) 
The second challenge is the computational cost due to the long-range feature of the electrostatic interaction.

A simple way to overcome these two challenges is to use a screened Coulombic interaction and another hard-core interaction to prevent overlap of the particles, as did in the work by Li et al. [156], but significant error may be caused by these assumptions. A screened Coulombic interaction is employed as

$\mathbf{F}_{i j}^{\text {Coulumb }}=\frac{q_{i} q_{j} l_{B} e^{-\kappa r_{i j}}}{r_{i j}}\left(\frac{1}{r_{i j}}+\kappa\right) \mathbf{n}_{i j}$,

where $l_{B}=e_{c}^{2} / k_{B} T \epsilon$ is the Bjerrum length, $\kappa$ is the inverse Debye length, and $q_{i}$ and $q_{j}$ are charges. In addition, a cut off length is defined so that this screened Coulombic interaction is only considered within this cut off distance. A hard-core Morse potential is applied to prevent ion clustering, which is given as

$\mathbf{F}_{i j}^{M}=2 D_{e} \alpha\left[e^{2 \alpha\left(2 r_{s}-r_{i j}\right)}-e^{\alpha\left(2 r_{s}-r_{i j}\right)}\right] \mathbf{n}_{i j}$,

where $D_{e}$ is the interaction coefficient, $\alpha$ characterizes the interaction range, and $r_{s}$ is the equilibrium length. Hard-core potential similar to Morse potential can be also used. For example, Yong et al. recently applied LJ potential to prevent ion clustering in studying the translocation time dependence on channel length [196]. However, since the advantage of DPD is that much larger time step can be used than MD because of its soft core interaction, this advantage may be diminished if a hard-core potential is employed in DPD.

Instead of using a hard-core potential, Groot applied the 'smeared charge' model to prevent the clustering of oppositely charged DPD particles [195]. The charge is distributed as a linear symmetric cloud with decreasing density as

$\rho(r)=\frac{3}{\pi r_{e}^{3}}\left(1-\frac{r}{r_{e}}\right)$,

where $r_{e}$ is the smearing radius and is usually set to $r_{e}=$ $1.6 r_{c}$, where $r_{c}$ is the cut off radius in DPD. The electrostatic potential between these two charge beads is approximately given by

$$
\begin{aligned}
& \frac{4 \pi r_{e} u(r)}{\Gamma r_{c}} \\
& = \begin{cases}\frac{52}{35}-\frac{4}{5}\left(\frac{r_{c}}{r_{e}}\right)^{2}+\frac{2}{5}\left(\frac{r r_{c}}{r_{e}}\right)^{4}-0.136\left(\frac{r_{c}}{r_{e}}\right)^{5.145}, & r<r_{e} / r_{c} \\
\frac{r_{e}}{r r_{c}}-3.21\left(1-\frac{r r_{c}}{2 r_{e}}\right)^{6}, & r_{e} / r_{c}<r<2 r_{e} / r_{c} \\
\frac{r_{e}}{r r_{c}}, & r>2 r_{e} / r_{c}\end{cases}
\end{aligned}
$$

where $\Gamma=e^{2} /\left(k_{B} T \epsilon_{0} \epsilon_{r} r_{c}\right)$ is the coupling constant, $e$ is the elementary charge, and $\epsilon_{0}$ and $\epsilon_{r}$ are absolute and relative electric permittivities. The charge is then applied on a grid, and the electrostatic field is solved locally by using a version of particle-particle particle-mesh (PPPM) algorithm by Becker et al. [197]. Yang et al. applied a similar method to study the translocation by modeling explicit counterions and the solvent quality in a computationally efficient way and considered two different driving forces [155].

Gonzalez-Melchor et al. [198] applied a Slater-type charge density to consider the charge distribution around a particle as

$\rho(r)=\frac{q}{\pi \lambda^{3}} e^{-2 r / \lambda}$,

where $\lambda$ is the decay length of the charge.

The corresponding interaction potential between two charged particles is given as

$\frac{4 \pi r_{e} u(r)}{\Gamma r_{c}}=\frac{1}{r}\left[1-(1+\beta r) e^{-2 \beta r}\right]$

where $\beta=r_{c} / \lambda$. To match Eq. 32 at $r=0$, we will have $\beta=52 /\left(35 r_{e}\right)$.

More importantly, they applied Ewald sum method to calculate the long-range interaction. Recently Wang et al. applied a non-uniform FFT-based Ewald summation method to calculate the long-range interaction based on Slater-type charge density in DPD simulations [199] and investigated the dendrimer-lipid interactions [200].

\subsubsection{Modeling of DNA/polymer translocation using lattice Boltzmann method}

Another popular methodology to model polymer translocation is to combine a coarse-grained model of particle dynamics [160] with Eulerian lattice Boltzmann (LB) fluid [201-205]. The particle and fluid can be coupled through the immersed boundary method (IB) or the bounce-back boundary condition [206-210]. The LB-IB method has been shown to capture the physical characteristics of polymer and particle deformation in shear flow, inter-particle hydrodynamic forces, and the near-wall hydrodynamic field [116, 118,202,207,211-213]. Direct comparisons with experimental measurements of DNA diffusivity and conformation in micro- and nanoslits have verified that LB accurately captures how DNA properties are affected by nano-confinement [136,214]. LB has also been shown to accurately predict DNA density distribution in microchannels [215].

In order for LB to resolve solvent hydrodynamic interactions, the time between solvent particle collisions must be very short compared to the time for fluid hydrodynamics to propagate in the system, so the mean free path of the solvent is much smaller than the system size, i.e., the Knudsen number $K n=\lambda / L<<1$, where $\lambda$ is the mean free path and $L$ is the representative physical length scale of the system. 
Microfluidic systems are well suited for LB modeling as the dominant length scale is $\mu \mathrm{m}$, while the mean free path of water molecules is on the scale of $\mathrm{nm}$. The validity of LB in the relevant parameter regimes is addressed in the context of a coarse-grained bead-spring model of DNA, which appears to satisfy that criterion. In addition, the Schmidt number $\left(S c=v / D_{m}\right)$, the ratio between the kinematic viscosity $v=\rho / \eta$ ( $\rho$ is the fluid density and $\eta$ is the fluid viscosity) and the coarse-grained bead diffusivity $D_{m}$, must be large so that the fluid propagates hydrodynamic interactions between beads much faster than the beads can diffuse. For molecules undergoing microfluidic flow, the competition between the inertial transport and viscous transport of the fluid is characterized by the Reynolds number $\operatorname{Re}=\rho U H / \eta$, which is usually very small, where $U$ is the flow velocity and $H$ is the channel dimension.

LB is carried out on a three-dimensional cubic lattice with 19 discrete velocities (the D3Q19 model). The lattice spacing is $\Delta x$, the kinematic viscosity is $\nu=\eta / \rho=1 / 6\left[\Delta x^{2} / \Delta \tau\right]$, and $\Delta \tau$ is the LB time step. Details of the LB method can be found in recent reviews [202,208,209,216,217]. The 19 discrete velocities $\mathbf{c}_{i}$ are given by the following vectors: $(0,0,0),( \pm 1,0,0),(0, \pm 1,0),(0,0, \pm 1),( \pm 1, \pm 1,0)$, $( \pm 1,0, \pm 1),(0, \pm 1, \pm 1)$. The magnitudes of the velocities are $c_{i}=\left|\mathbf{c}_{i}\right|=0,1, \sqrt{2}$. The lattice simulation has grid size $\Delta x$ and time step $\Delta t$. The maximum velocity in the simulation is the speed of sound $c_{s}=\Delta x / \Delta t$. The velocity distribution function $n_{i}(\mathbf{r}, t)$ gives the fluid population with the velocity $\mathbf{c}_{i}$ at position $\mathbf{r}$ at time $t$. At equilibrium, the velocity distribution functions can be represented as a second-order expansion of the Maxwell-Boltzmann distribution, given by

$$
\begin{aligned}
& n_{i}^{e q}(\mathbf{r}, t)=\rho a^{c_{i}}\left(1+\left(\mathbf{c}_{i} \cdot \mathbf{u}\right) / c_{s}^{2}\right. \\
& \left.+\mathbf{u u}:\left(\mathbf{c}_{i} \mathbf{c}_{i}-c_{s}^{2} \mathbf{I}\right) /\left(2 c_{s}^{4}\right)\right), \\
& \rho=\sum n_{i}^{e q}, \\
& \rho \mathbf{u}=\sum \mathbf{c}_{i} \cdot n_{i}^{e q}, \\
& \Pi=\rho\left(\mathbf{u u}+c_{s}^{2} \mathbf{I}\right)=\sum n_{i}^{e q} \cdot \mathbf{c}_{i} \mathbf{c}_{i}, \\
& \sum a^{c_{i}} c_{i \alpha} c_{i \beta} c_{i \gamma} c_{i \delta}=c_{s}^{4}\left(\delta_{\alpha \beta} \delta_{\gamma \delta}+\delta_{\alpha \gamma} \delta_{\beta \delta}+\delta_{\alpha \delta} \delta_{\beta \gamma}\right) \text {. }
\end{aligned}
$$

The indices $\alpha, \beta, \gamma$, and $\delta$ denote axis $\mathrm{x}, \mathrm{y}$, and $\mathrm{z}$. $\mathbf{u}$ is the local velocity and the coefficients $a^{c_{i}}$ are determined by satisfying the equilibrium conditions and the isotropy condition in Eq. 35. For the D3Q19 system, $a^{0}=1 / 3, a^{1}=$ $1 / 18, a^{\sqrt{2}}=1 / 36$. In terms of the fluid hydrodynamic properties, the velocity distribution functions are transformed to the hydrodynamic moments, $\mathbf{M}_{q}=\mathbf{m} \cdot \mathbf{n}$, where $\mathbf{M}_{q}$ is the $q$ th moment of $n=\left(n_{0}, n_{1}, \ldots, n_{18}\right)$, and $\mathbf{m}$ is the transformation matrix. The 19 moments of the velocity distribution function are the density $\rho$, the momentum density $\mathbf{j}=\rho \mathbf{u}$, the momentum flux $\Pi=\rho\left(\mathbf{u u}+c_{s}^{2} \mathbf{I}\right)$, and the kinetic energy flux that conserve energy ('ghost' moments). At each time step, the fluid particles undergo local collision at the lattice site, and the velocity distribution functions evolve as

$n_{i}\left(\mathbf{r}+\mathbf{c}_{i} \mathrm{~d} t, t+\mathrm{d} t\right)=n_{i}(\mathbf{r}, t)+L_{i j}\left[n_{j}(\mathbf{r}, t)-n_{j}^{e q}(\mathbf{r}, t)\right]$,

where $L$ is a collision operator for dissipation due to fluid particle collisions such that the fluid always relaxes toward the equilibrium distribution. Local dissipation of the fluid momentum is justified if the particle mean free path is much shorter than the lattice size, i.e., $K n<<1$. For small $\mathrm{Kn}$ and Mach number, Eq. 35 has been shown to be equivalent to the Navier-Stokes equation.

Hydrodynamic interactions between the DNA segments can be captured with the exchange and propagation of frictional momentum between the fluid and the polymer [202, 213]. Each bead on the polymer experiences a friction force

$\mathbf{F}_{f}=-\zeta\left[\mathbf{u}_{b}\left(\mathbf{r}_{b}\right)-\mathbf{u}_{f}\left(\mathbf{r}_{b}\right)\right]$,

where $\mathbf{u}_{b}\left(\mathbf{r}_{b}\right)$ and $\mathbf{u}_{f}\left(\mathbf{r}_{b}\right)$ are the bead and fluid velocity at the bead position $\mathbf{r}_{b}$, respectively, and $\zeta$ is the bead friction coefficient. The fluid velocity at position $\mathbf{r}_{b}$ is determined by a trilinear interpolation of the fluid velocity on the neighboring lattice points $n n$ that enclose $\mathbf{r}_{b}, \mathbf{u}_{f}\left(\mathbf{r}_{b}\right)=\sum_{\mathbf{r} \in n n} w_{\mathbf{r}} \mathbf{u}(\mathbf{r})$, where the weights $w_{\mathbf{r}}$ are the coefficients of the normalized linear Lagrange interpolation polynomial. The frictional momentum density $\Delta \mathbf{j}=-\mathbf{F}_{f} \delta t / \Delta x^{3}$ is transferred to the fluid.

With the assumptions involved in LB and IB, systematic errors can arise due to fluid compressibility and the momentum transfer between the particle and the fluid. With the immersed boundary method, the no-slip boundary condition is imposed on the particle surface, and the friction momentum is calculated based on first-order Lagrange interpolation of the fluid velocity at the bead position from the lattice fluid velocity. Thus, the accuracy of momentum coupling is of $\mathrm{O}\left(\Delta x^{2}\right)$. Furthermore, the fluid velocity from LB is of $\mathrm{O}\left(\Delta x^{2}\right)$ accuracy.

Reboux et al. applied a coarse-grained model to solve the electrokinetic equations at the Poisson-Boltzmann level for the microions and coupled it to a LB equation for the solvent hydrodynamics to study DNA translocation [218]. Fyta et al. studied the problem of polymer fast translocation through a nanopore [151]. Melchionna et al. investigated the probability distributions of the translocation time of the DNA through the nanopore by adopting various initial configurations and lengths [219,220]. Izmitli studied the electric-field driven translocation of 21-210 $\mu \mathrm{m}$ DNA with and without hydrodynamic interactions and found that the effect of hydrodynamic interactions is local and the initial configuration of 
the molecules is much more important in determining the dynamics of DNA is for fast translocation processes [172]. It was also found that the coupling of the correlated molecular motion to hydrodynamics causes significant acceleration of the translocation process [152], and wide pores can host a larger number of multiple biopolymer segments, as compared to smaller pores [221]. In addition, Alapati et al. found that translocation velocity mainly depends upon the applied potential difference rather than upon the electric field inside the nanopores [222-224].

\section{Challenges and future directions}

Although both RBCs and DNAs have been studied extensively using various computational methods, some important questions still remain unanswered, especially due to the correlation between physical and chemical processes in various length scales. For example, the stress-free shape of RBCs is not completely understood [53], and more experiments are required to quantify the exact stress-free state of RBCs, which is closely related to the biconcave resting shape [26,54], which is the first of eight mysteries about RBC proposed by Hoffman [228]. More importantly, how the membrane protein mutations lead to RBC diseases such as hereditary spherocytosis and elliptocytosis remain unclear. This problem has been explored using coarse-grained MD for a small piece of membrane [30,31], but currently it is computationally prohibitive to extend this model to study a whole RBC. It is also a challenge to simulate the bilayer vesiculation process in RBC aging and RBC diseases using existing models, since it involves topological changes of the lipid bilayer. It can be done in a small scale [31], but not in the cell scale. Furthermore, chemical reaction processes such as binding of ATP to the cytoskeleton [229] and polymerization of abnormal hemoglobin in sickle cell disease [230-232] have not been accurately connected to the mechanical models yet. It is critical to connect biophysical models and chemical kinetics models for a better understanding of RBC physiology, such as RBC's role in relaxing capillaries for improved blood perfusion.

For modeling of DNA translocation through nanopore, one of the critical issues is to identify the variation of electric current signature for different base pairs for the purpose of sequencing. Although mesoscale modeling approaches such as DPD and LB can be used to capture DNA dynamics on length scales of a few nanometers, several challenges remain. The most obvious of which is the high computational cost of modeling even small systems. Advances in algorithm, parallelization, or GPU-accelerated methods may be explored to reduce the computational time. Another challenge is in determining how to incorporate sufficient molecular details while maintain the advantages of coarse-grained modeling.
Computational modeling research will continue to test the predictions of different levels of molecular coarse-graining to validate the model predictions. In addition, a common difficulty for both DPD and LB is to match the ratio between the fluid momentum diffusion and the coarse-grained bead diffusion (Schmidt number $S c$ ) between the coarse-grained and the molecular systems. In the case of DPD, the Schmidt number is about 1000 times lower than the actual physical value of water. This may introduce computational artifacts in the simulation of a dynamic nonequilibrium process such as DNA translocation through a nanopore using DPD and LB. As with all computational results of real systems, results and predictions need to be thoroughly and rigorously tested against real observations. Despite these challenges, important new insights of dynamic mechanisms may be gained from the coarse-grained modeling of DNA and RBC for the appropriately chosen systems. Finally, although coarsegrained simulations is capable of predicting the translocation dynamics and providing guidance for the appropriate parameters for conductivity measurements, accurate calculations of the single nucleotide conductivity are still needed in order to differentiate between different bases. Future advances will require the combination of coarse-grained model of molecular configurations during translocation with first-principle calculations of nucleotide conductivity in order to provide model predictions of conductivity during translocation.

Even as the computational capacities keep increasing, mesoscale models will continue to provide a bridge between continuum and atomistic methods to fill the gap in our understanding of molecular dynamics and material properties. The successful examples of RBC and DNA have demonstrated the capabilities of mesoscale coarse-grained modeling as a tool of understanding cellular and biomolecular dynamics.

\section{References}

1. Walpole J, Papin JA, Peirce SM (2013) Multiscale computational models of complex biological systems. Annu Rev Biomed Eng 15:137-154

2. Shaw D (2007) Anton, a special-purpose machine for molecular dynamics simulation. ACM Press, New York, pp 1-12

3. Nobel Media AB (2014) The Nobel Prize in Chemistry 2014 (http://www.nobelprize.org/nobel_prizes/chemistry/ laureates/2014/)

4. Bao G, Suresh S (2003) Cell and molecular mechanics of biological materials. Nat Mater 2:715-725

5. Nobel Media AB (2013) The Nobel Prize in Chemistry 2013 (http://www.nobelprize.org/nobel_prizes/chemistry/ laureates/2013/)

6. Weinan E (2011) Principles of multiscale modeling. Cambridge Press, Cambridge

7. Hughes T (2000) The finite element method: linear static and dynamic finite element analysis. Dover Publications, New York 
8. Belytschko T, Liu W, Moran B (2000) Nonlinear finite elements for continua and structures. Wiley, New York

9. Pozrikidis C (1992) Boundary integral and singularity methods for linearized viscous flow. Cambridge University Press, New York

10. Morton KW, Mayers DF (2005) Numerical solution of partial differential equations, an introduction. Cambridge University Press, Cambridge

11. LeVeque R (2002) Finite volume methods for hyperbolic problems. Cambridge University Press, Cambridge

12. Karniadakis G, Sherwin S (2005) Spectral/hp element methods for computational fluid dynamics, 2nd edn. Oxford University Press, Oxford

13. Fung YC (1993) Biomechanics: mechanical properties of living tissues. Springer, New York

14. Evans E, Skalak P (1980) Mechanics and thermodynamics of biomembranes. CRC Press, Boca Raton

15. Humphrey JD (2002) Cardiovascular solid mechanics: cells, tissues, and organs. Springer Science \& Business Media, New York

16. Karplus M, McCammon JA (2002) Molecular dynamics simulations of biomolecules. Nat Struct Biol 9:646-652

17. Walensky LD, Mohandas N, Lux SE (2003) Disorders of the red blood cell membrane. In: Handin RI, Lux SE, Stossel TP (eds) Blood: principles and practice of hematology, 2nd edn. Lippincott Williams \& Wilkins, Philadelphia, pp 1709-1858

18. Li X, Vlahovska P, Karniadakis G (2013) Continuum- and particle-based modeling of shapes and dynamics of red blood cells in health and disease. Soft Matter 9:28

19. Peng Z, Li X, Pivkin IV, Dao M, Karniadakis GE, Suresh S (2013) Lipid bilayer and cytoskeletal interactions in a red blood cell. Proc Natl Acad Sci USA 110:13356-13361

20. Dao M, Lim CT, Suresh S (2003) Mechanics of the human red blood cell deformed by optical tweezers. J Mech Phys Solids 51:2259-2280

21. Peng Z, Asaro R, Zhu Q (2010) Multiscale simulation of erythrocyte membranes. Phys Rev E 81:031904

22. Peng Z, Asaro R, Zhu Q (2011) Multiscale simulation of erythrocyte membranes. J Fluid Mech 686:299-337

23. Boey SK, Boal DH, Discher DE (1998) Simulations of the erythrocyte cytoskeleton at large deformation. II. Micropipette aspiration. Biophys J 75:1584-1597

24. Li J, Dao M, Lim CT, Suresh S (2005) Spectrin-level modeling of the cytoskeleton and optical tweezers stretching of the erythrocyte. Biophys J 88:3707

25. Pivkin IV, Karniadakis GE (2008) Accurate coarse-grained modeling of red blood cells. Phys Rev Lett 101:118105

26. Peng Z, Mashayekh A, Zhu Q (2014) Erythrocyte responses in low shear rate flows - effects of non-biconcave stress-free state in cytoskeleton. J Fluid Mech 742:96-118

27. Noguchi H, Gompper G (2005) Shape transitions of fluid vesicles and red blood cells in capillary flows. Proc Natl Acad Sci USA 102:14159-14164

28. Zhu Q, Vera C, Asaro R, Sche P, Sung L (2007) A hybrid model for erythrocyte membrane: a single unit of protein network coupled with lipid bilayer. Biophys J 93:386-400

29. Zhu Q, Asaro R (2008) Spectrin folding vs. unfolding reactions and RBC membrane stiffness. Biophys J 94:2529-2545

30. Li H, Lykotrafitis G (2012) Two-component coarse-grained molecular-dynamics model for the human erythrocyte membrane. Biophys J 102:75-84

31. Li H, Lykotrafitis G (2014) Erythrocyte membrane model with explicit description of the lipid bilayer and the spectrin network. Biophys J 107:642-653

32. Li J, Lykotrafitis G, Dao M, Suresh S (2007) Cytoskeletal dynamics of human erythrocyte. Proc Natl Acad Sci USA 104:49374942
33. Alberts B, Johnson A, Lewis J, Raff M, Roberts K, Walter P (2002) Molecular biology of the cell. Garland Science, New York

34. Waugh R, Narla M, Jackson C, Mueller T, Suzuki T, Dale G (1992) Rheologic properties of senescent erythrocytes: loss of surface area and volume with red blood cell age. Blood 79:1351-1358

35. Peng Z, Zhu Q (2013) Deformation of the erythrocyte cytoskeleton in tank treading motions. Soft Matter 9:7617-7627

36. Bagchi P, Kalluri R (2009) Dynamics of nonspherical capsules in shear flow. Phys Rev E 80:016307

37. Yazdani A, Bagchi P (2011) Phase diagram and breathing dynamics of a single red blood cell and a biconcave capsule in dilute shear flow. Phys Rev E 84:026314

38. Zhao H, Isfahania A, Olsonc L, Freund J (2010) A spectral boundary integral method for flowing blood cells. J Comput Phys 229:3726

39. Freund J (2014) Numerical simulation of flowing blood cells. Annu Rev Fluid Mech 46:67-95

40. Fischer TM, Stohr-Liesen M, Schmid-Schonbein H (1978) The red cell as a fluid droplet: tank tread-like motion of the human erythrocyte membrane in shear flow. Science 202:894-896

41. Tran-Son-Tay R, Sutera S, Rao P (1984) Determination of red blood cell membrane viscosity from rheoscopic observations of tank-treading motion. Biophys J 46:65-72

42. Lee JC, Discher DE (2001) Deformation-enhanced fluctuations in the red cell skeleton with theoretical relations to elasticity, connectivity, and spectrin unfolding. Biophys J 81:3178-3192

43. Rief M, Pascual J, Saraste M, Gaub HE (1999) Single molecule force spectroscopy of spectrin repeats: low unfolding forces in helix bundles. J Mol Biol 286:553

44. Sung LA, Vera C (2003) Protofilament and hexagon: a threedimensional mechanical model for the junctional complex in the RBC membrane skeleton. Ann Biomed Eng 31:1314-1326

45. Fischer T (1992) Is the surface area of the red cell membrane skeleton locally conserved? Biophys J 61:298

46. Dodson RW, Dimitrakopoulos P (2010) Tank-treading of erythrocytes in strong shear flows via a nonstiff cytoskeleton-based continuum computational modeling. Biophys J 99:2906-2916

47. Malone JG, Johnson NL (1994) A parallel finite-element contact/impact algorithm for nonlinear explicit transient analysis 1 . The search algorithm and contact mechanics. Int J Num Methods Eng 37:559-590

48. Kapitza H, Rupped D, Galla H, Sackmann E (1984) Lateral diffusion of lipids and glycophorin in solid phosphatidylcholine bilayers. The role of structural defects. Biophys J 45:577

49. Kodippili G, Spector J, Sullivan C, Kuypers F, Labotka R, Gallagher P, Ritchie K, Low P (2009) Imaging of the diffusion of single band 3 molecules on normal and mutant erythrocytes. Blood 113:6237

50. Tomishige M (1998) Regulation mechanism of the lateral diffusion of band 3 in erythrocyte membranes by the membrane skeleton. J Cell Biol 142:989-1000

51. Fedosov DA, Caswell B, Karniadakis GE (2011) Wall shear stressbased model for adhesive dynamics of red blood cells in malaria. Biophys J 100:2084-2093

52. Walter J, Salsac A, Barthès-Biesel D, Tallec PL (2010) Coupling of finite element and boundary integral methods for a capsule in a stokes flow. Int J Num Methods Eng 83:829

53. Dupire J, Socol M, Viallat A (2012) Full dynamics of a red blood cell in shear flow. Proc Natl Acad Sci USA 109:20808-20813

54. Peng Z, Salehyar S, Zhu Q (2015) Stability of the tank treading modes of erythrocytes and its dependence on cytoskeleton reference states. J Fluid Mech 771:449-467

55. Knowles DW, Tilley L, Mohandas N, Chasis JA (1997) Erythrocyte membrane vesiculation: model for the molecular mechanism of protein sorting. Proc Natl Acad Sci USA 94:12969-12974 
56. Hoogerbrugge PJ, Koelman JMVA (1992) Simulating microscopic hydrodynamic phenomena with dissipative particle dynamics. Europhys Lett 19:155-160

57. Groot RD, Warren PB (1997) Dissipative particle dynamics: bridging the gap between atomistic and mesoscopic simulation. J Chem Phys 107:4423-4435

58. Qiao R, He P (2007) Mapping of dissipative particle dynamics in fluctuating hydrodynamics simulations. J Chem Phys 128:126101

59. Berk DA, Hochmuth RM, Waugh RE (1989) In: Agre P, Parker JC (eds) Viscoelastic properties and rheology. Red blood cell membranes. Marcel Dekker, New York, pp 445-446

60. Heinrich V, Ritchie K, Mohandas N, Evans E (2001) Elastic thickness compressibilty of the red cell membrane. Biophys $\mathrm{J}$ 81:1452-1463

61. Espanol P (1998) Fluid particle model. Phys Rev E 57:2930-2948

62. Discher D, Mohandas N, Evans E (1994) Molecular maps of red cell deformation: hidden elasticity and in situ connectivity. Science 266:1032-1035

63. Evans EA, Waugh R (1979) Thermoelasticity of red blood cell membrane. Biophys J 26:115-131

64. Evans EA, Waugh R, Melnik L (1976) Elastic area compressibility modulus of red-cell membrane. Biophys J 16:585-595

65. Park Y, Diez-Silva M, Popescu G, Lykotrafitis G, Choi W, Feld MS, Suresh S (2008) Refractive index maps and membrane dynamics of human red blood cells parasitized by Plasmodium falciparum. Proc Natl Acad Sci USA 105:13730-13735

66. Fischer TM (2007) Tank-tread frequency of the red cell membrane: dependence on the viscosity of the suspending medium. Biophys J 93:2553-2561

67. Hochmuth R, Mohandas N, Blackshear P (1973) Measurement of the elastic modulus for red cell membrane using a fluid mechanical technique. Biophys J 13:747-762

68. Li X, Peng Z, Lei H, Dao M, Karniadakis G (2014) Probing the RBC dynamics, rhelogy and mechnics with the two component cell model. Phil Trans R Soc A 372:2021

69. Zhang Y, Huang C, Kim S, Golkaram M, Dixon M, Tilley L, Li J, Zhang S, Suresh S (2015) Multiple stiffening effects of nanoscale knobs on human red blood cells infected with Plasmodium falciparum malaria parasite. Proc Natl Acad Sci USA 112:60686073

70. Reasor DA, Clausen JR, Aidun CK (2012) Coupling the latticeBoltzmann and spectrin-link methods for the direct numerical simulation of cellular blood flow. Int J Num Methods Fluids 68:767

71. Zhang JF, Johnson PC, Popel AS (2008) Red blood cell aggregation and dissociation in shear flows simulated by lattice Boltzmann method. J Biomech 41:47-55

72. Ramanujan S, Pozrikidis C (1998) Deformation of liquid capsules enclosed by elastic membranes in simple shear flow: large deformations and the effect of fluid viscosities. J Fluid Mech 361:117-143

73. Dupont C, Salsac AV, Barthès-Biesel D (2013) Off-plane motion of a prolate capsule in shear flow. J Fluid Mech 721:180-198

74. Freund $\mathbf{J}$ (2013) The flow of red blood cells through a narrow spleen-like slit. Phys Fluids 25:110807

75. Yuan H, Huang C, Li J, Lykotrafitis G, Zhang S (2010) Oneparticle-thick, solvent-free, coarse-grained model for biological and biomimetic fluid membranes. Phys Rev E 82:011905

76. Fedosov D, Peltomaki M, Gompper G (2014) Deformation and dynamics of red blood cells in flow through cylindrical microchannels. Soft Matter 10:4258-4267

77. Muller K, Fedosov D, Gompper G (2015) Smoothed dissipative particle dynamics with angular momentum conservation. J Comput Phys 281:301-315

78. Hosseini S, Feng J (2012) How malaria parasites reduce the deformability of infected red blood cells. Biophy J 103:1-10
79. Van Liedekerke P, Odenthal T, Smeets B, Ramon H (2013) Solving microscopic flow problems using Stokes equations in SPH. Comput Phys Commun 184:1686-1696

80. Witthoft A, Yazdani A, Peng Z, Bellini C, Humphrey J, Karniadakis $\mathrm{G}$ (2015) A discrete particle model of a multilayered fiber-reinforced arterial wall. J R Soc Interface submitted

81. Boal D (2012) Mechanics of the cell, 2nd edn. Cambridge University Press, Cambridge

82. Marko JF, Siggia ED (1994) Stretching DNA. Macromolecules 28:8759

83. Shaqfeh ESG (2004) The dynamics of single molecule DNA in flow. J Non-Newtonian Fluid Mech 130:2005

84. Usta O, Ladd AJC, Butler JE (2005) Lattice-boltzmann simulations of the dynamics of polymer solutions in periodic and confined geometries. J Chem Phys 122:094902

85. Watari N, Makino M, Kikuchi N, Larson RG, Doi M (2007) Simulation of DNA motion in a microchannel using stochastic rotation dynamics. J Chem Phys 126:094902

86. de Pablo JJ (2011) Coarse-grained simulations of macromolecules: from DNA to nanocomposites. Annu Rev Phys Chem 62:555-574

87. Bustamante C, Bryant Z, Smith SB (2003) Ten years of tension: single-molecule DNA mechanics. Nature 421:423

88. Jendrejack RM, Dimalanta ET, Schwartz DC, Graham MD, de Pablo JJ (2003) DNA dynamics in a microchannel. Phys Rev Lett 91:038102

89. Hegde GA, Chang J-F, Chen Y-L, Khare R (2011) Conformation and diffusion behavior of ring polymers in solution. J Chem Phys 135:184901

90. Doyle PS, Shaqfeh ESG, McKinley GH, Spiegelberg SH (1998) Relaxation of dilute polymer solutions following extensional flow. J Non-Newtonian Fluid Mech 76:79-110

91. Hur JS, Shaqfeh ESG, Larson RG (2000) Brownian dynamics simulations of single DNA molecules in shear flow. J Rheol 44:713-742

92. Schroeder CM, Teixeira RE, Shaqfeh ESG, Chu S (2005) Dynamics of DNA in the flow-gradient plane of steady shear flow: observations and simulations. Macromolecules 38:1967-1978

93. Müller-Plathe F (2002) Coarse-graining in polymer simulation: from the atomistic to the mesoscopic scale and back. Chem Phys Chem 3:754-769

94. Kreft J, Chen YL, Chang HC (2008) Conformation and trapping rate of DNA at a convergent stagnation point. Phys Rev E 77:030801

95. Teclemariam NP, Beck VA, Shaqfeh ESG, Muller SJ (2007) Dynamics of DNA polymers in post arrays: comparison of single molecule experiments and simulations. Macromolecules 40:3848-3859

96. Hsu H-P, Grassberger P (2004) Polymers confined between two parallel plane walls. J Chem Phys 120:2034

97. Bleha T, Cifra P (2003) Free energy and confinement force of macromolecules in a slit at full equilibrium with a bulk solution. Polymer 44:3745-3752

98. Chaudhuri D, Mulder B (2011) Size and shape of excluded volume polymers confined between parallel plates. Phys Rev E 83:031803

99. Cui T, Ding JD, Chen JZY (2008) Dynamics of a self-avoiding polymer chain in slit, tube, and cube confinements. Phys Rev E 78:061802

100. Odijk T (2008) Scaling theory of DNA confined in nanochannels and nanoslits. Phys Rev E 77:060901 (R)

101. Cifra P (2012) Weak-to-strong confinement transition of semiflexible macromolecules in slit and in channel. J Chem Phys 136:024902

102. Dai L, Jones JJ, van der Maarel JRC, Doyle PS (2012) A systematic study of DNA conformation in slitlike confinement. Soft Matter 8:2972 
103. Dai L, Tree DR, van der Maarel JRC, Dorfman KD, Doyle PS (2013) Revisiting blob theory for DNA diffusivity in slitlike confinement. Phys Rev Lett 110:168105

104. Chen YL, Graham MD, de Pablo J, Randall GC, Gupta M, Doyle PS (2004) Conformation and dynamics of single DNA molecules in parallel-plate slit microchannels. Phys Rev E 70:060901

105. Chen JZY, Sullivan DE (2006) Free energy of a wormlike polymer chain confined in a slit: crossover between two scaling regimes. Macromolecules 39:7769

106. Chen Y-L, Lin PK, Chou CF (2010) Generalized force-extension relation for wormlike chains in slit confinement. Macromolecules 43:10204

107. Lin PK, Hsieh CC, Chen YL, Chou CF (2012) Effects of topology and ionic strength on double-stranded DNA confined in nanoslits. Macromolecules 45:2920-2927

108. Ollila STT, Denniston C, Karttunen M, Ala-Nissila T (2013) Hydrodynamic effects on confined polymers. Soft Matter 9:34783487

109. Tree DR, Reinhart WF, Dorfman KD (2014) The Odijk regime in slits. Macromolecules 47:3672-3684

110. Yeh JW, Taoni A, Chen YL, Chou CF (2012) Entropy-driven single molecule Tug-of-war of DNA at micro-nanofluidic interfaces. Nano Lett 12:1597-1602

111. Zhu Q, Zeng J, Triantafyllou MS, Yue DKP (2006) Direct numerical simulation of single molecule-DNA by cable dynamics. J MEMS 15:1078-1087

112. Daoud M, deGennes PG (1977) Statistics of macromolecular solutions trapped in small pores. J Phys (Paris) 38:85

113. Brochard F, de Gennes PG (1977) Dynamics of confined polymer chains. J Chem Phys 67:52

114. Odijk T (1983) On the statistics and dynamics of confined or entangled stiff polymers. Macromolecules 16:1340

115. Jendrejack RM, Schwartz DC, Graham MD, de Pablo JJ (2003) Effect of confinement on DNA dynamics in microfluidic devices. J Chem Phys 119:1165

116. Usta OB, Butler JE, Ladd AJC (2006) Flow-induced migration of polymers in dilute solution. Phys Fluids 18:031703

117. Chen Y-L, Jo K, Graham MD, Schwartz DC, de Pablo JJ (2005) DNA molecules in microfluidic oscillatory flow. Macromolecules 38:6680

118. Chen Y-L, Ma H, Graham MD, De Pablo JJ (2007) Modeling DNA in confinement: a comparison between the brownian dynamics and lattice boltzmann method. Macromolecules 40:5978

119. Burkhardt TW, Yang Y, Gompper G (2010) Fluctuations of a long, semiflexible polymer in a narrow channel. Phys Rev E 82:041801

120. Burkhardt T (1995) Free energy of a semiflexible polymer confined along an axis. J Phys A: Math Gen 28:L629

121. Chen Y-L (2013) Electro-entropic excluded volume effects on DNA looping and relaxation in nanochannels. Biomicrofluidics 7:054119

122. Tree DR, Wang Y, Dorfman KD (2012) Mobility of a semiflexible chain confined in a nanochannel. Phys Rev Lett 108:2208105

123. Tree DR, Wang Y, Dorfman KD (2013) Modeling the relaxation time of DNA confined in a nanochannel. Biomicrofluidics 7:054118

124. Woo NJ, Shaqfeh ESG, Khomami B (2004) Effect of confinement on dynamics and rheology of dilute deoxyribose nucleic acid solutions. II. Effective rheology and single chain dynamics. J Rheol 48:299

125. Perkins TT, Quake SR, Smith DE, Chu S (1994) Relaxation of a single DNA molecule observed by optical microscopy. Science 264:822-826

126. Smith DE, Perkins TT, Chu S (1996) Dynamical scaling of DNA diffusion coefficients. Macromolecules 29:1372

127. Smith DE, Babcock HP, Chu S (1999) Single-polymer dynamics in steady shear flow. Science 283:1724
128. Maier B, Rdler JO (1999) Conformation and self-diffusion of single DNA molecules confined to two dimensions. Phys Rev Lett 82:1911

129. Tang J, Levy SL, Trahan DW, Jones JJ, Craighead HG, Doyle PS (2010) Revisiting the conformation and dynamics of DNA in slitlike confinement. Macromolecules 43:7368

130. Strychalski EA, Levy SL, Craighead HG (2008) Diffusion of DNA in nanoslits. Macromolecules 41:7716-7721

131. Strychalski EA, Geist J, Gaitan M, Locasio LE, Stavis SM (2012) Quantitative measurements of the size scaling of linear and circular DNA in nanofluidic slitlike confinement. Macromolecules 45:1602

132. Stavis SM, Geist J, Gaitan M, Locascio LE, Strychalski EA (2012) DNA molecules descending a nanofluidic staircase by entropophoresis. Lab Chip 12:1174

133. Cross JD, Strychalski EA, Craighead HG (2007) Size-dependent DNA mobility in nanochannels. J Appl Phys 102:024701

134. Reisner W, Morton KJ, Riehn R, Wang YM, Yu Z, Rosen M, Sturm JC, Chou SY, Frey E, Austin RH (2005) Statics and dynamics of single DNA molecules confined in nanochannels. Phys Rev Lett 94:196101

135. Reisner W, Beech JP, Larsen NB, Flyvbjerg H, Kristensen A, Tegenfeldt JO (2007) Nanoconfinement-enhanced conformational response of single DNA molecules to changes in ionic environment. Phys Rev Lett 99:058302

136. Lin P-K, Chang J-F, Wei CH, Tsao PH, Fann WS, Chen Y-L (2011) Partial hydrodynamic screening of confined linear and circular double stranded DNA dynamics. Phys Rev E 84:031917

137. Hsieh C-C, Balducci A, Doyle PS (2007) An experimental study of DNA rotational relaxation time in nanoslits. Macromolecules 40:5196

138. Persson F, Utko P, Reisner W, Larsen NB, Kristensen A (2009) Confinement spectroscopy: probing single DNA molecules with tapered nanochannels. Nano Lett 9:1382

139. Lin P-K, Fu C-C, Chen Y-L, Chen Y-R, Wei P-K, Kuan CH, Fann WS (2007) Static conformation and dynamics of single DNA molecules confined in nanoslits. Phys Rev E 76:011806

140. Kim Y, Kim KS, Kounovsky KL, Chang R, Jung GY, dePablo JJ, Jo K, Schwartz DC (2011) Nanochannel confinement, DNA stretch approaching full contour length. Lab Chip 11:1721

141. Balducci A, Mao P, Han J, Doyle PS (2006) Double-stranded DNA diffusion in slitlike nanochannels. Macromolecules 39:6273

142. Witz G, Rechendorff K, Adamcik J, Dietler G (2008) Conformation of circular DNA in two dimensions. Phys Rev Lett $101: 148103$

143. Tree DR, Muralidhar A, Doyle PS, Dorfman KD (2013) Extension of DNA in a nanochannel as a rod-to-coil transition. Macromolecules 43:8369

144. Mansfield ML, Douglas JF (2013) Is duplex DNA a swollen random coil? Soft Matter 9:8914-8922

145. Reisner W, Larsen NB, Silahtaroglu A, Kristensen A, Tommerup N, Tegenfeldt JO, Flyvbjerg H (2010) Single-molecule denaturation mapping of DNA in nanofluidic channels. Proc Natl Acad Sci USA 107:13294-13299

146. Reisner W, Larsen NB, Flyvbjerg H, Tegenfeldt JO, Kristensen A (2009) Directed self-organization of single DNA molecules in a nanoslit via embedded nanopit arrays. Proc Natl Acad Sci USA 106:79-84

147. Jo K, Dhingra DM, Odijk T, De Pablo JJ, Graham MD, Runnheim R, Forrest D, Schwartz DC (2007) A single-molecule barcoding system using nanoslits for DNA analysis. Proc Natl Acad Sci USA 104:2673

148. Dimalanta ET, Lim A, Runnheim R, Lamers C, Churas C, Forrest DK, de Pablo JJ, Graham MD, Coppersmith SN, Goldstein S, Schwartz DC (2004) A microfluidic system for large DNA molecule arrays. Anal Chem 76:5293-301 
149. Austin RH, Tegeneldt JO, Cao H, Chou SY, Cox EC (2002) Scanning the controls: genomics and nanotechnology. IEEE Trans Nanotech 1:12

150. Sauer M, Angerer B, Ankenbauer W, Fldes-Papp Z, Gbel F, Han K-T, Rigler R, Schulz A, Wolfrum J, Zander C (2001) Single molecule DNA sequencing in submicrometer channels: state of the art and future prospects. J Biotechnol 86:181

151. Fyta MG, Melchionna S, Kaxiras E, Succi S (2006) Multiscale coupling of molecular dynamics and hydrodynamics: application to DNA translocation through a nanopore. Multiscale Model Simul 5:1156-1173

152. Fyta MG, Melchionna S, Succi S, Kaxiras E (2006) Hydrodynamic correlations in the translocation of a biopolymer through a nanopore: theory and multiscale simulations. Phys Rev E 78:036704

153. Hernndez-Ortiz J-P, de Pablo J, Graham M (2007) Fast computation of many-particle hydrodynamic and electrostatic interactions in a confined geometry. Phys Rev Lett 98:140602

154. Hernndez-Ortiz J-P, Graham MD, De Pablo JJ (2006) N log N method for hydrodynamic interactions of confined polymer systems: Brownian dynamics. J Chem Phys 125:164906

155. Yang K, Vishnyakov A, Neimark AV (2013) Polymer translocation through a nanopore: DPD study. J Phys Chem B 117:36483658

156. Li XJ, Li XL, Deng MG, Liang HJ (2012) Electrostatic interactions on the translocation of polymers through a narrow pore under different solvent conditions: a dissipative particle dynamics simulation study. Macromol Theory Simul 21:120-129

157. Malevanets A, Kapral R (1999) Mesoscopic model for solvent dynamics. J Chem Phys 110:8605-8613

158. Jendrejack RM, Schwartz DC, Graham MD, de Pablo JJ (2003) Effect of confinement on DNA dynamics in microfluidic devices. J Chem Phys 119:1165-1173

159. Hsieh C-C, Li L, Larson RG (2003) Modeling hydrodynamic interaction in Brownian dynamics: simulations of extensional flows of dilute solutions of DNA and polystyrene. J NonNewtonian Fluid Mech 113:147-191

160. Allen MP, Tildesley DJ (1987) Computer simulation of liquids. Clarendon Press, Oxford

161. Rubinstein M, Colby RH (2003) Polymer physics. Oxford University Press Inc., New York

162. Underhill PT, Doyle PS (2004) On the coarse-graining of polymers into bead-spring chains. J Non-Newtonian Fluid Mech 122:3

163. Knotts TA IV, Rathore N, Schwartz DC, de Pablo JJ (2007) A coarse grain model for DNA. J Chem Phys 126:084901

164. Hinckley DM, Freeman GS, Whitmer JK, de Pablo JJ (2013) An experimentally-informed coarse-grained 3-Site-Per-Nucleotide model of DNA: structure, thermodynamics, and dynamics of hybridization. J Chem Phys 139:144903

165. Branton D, Deamer DW, Marziali A, Bayley H, Benner SA, Butler T, Di Ventra M, Garaj S, Hibbs A, Huang X et al (2008) The potential and challenges of nanopore sequencing. Nat Biotechnol 26:1146-1153

166. Lagerqvist J, Zwolak M, Di Ventra M (2006) Fast DNA sequencing via transverse electronic transport. Nano Lett 6:779-782

167. Meller A, Nivon L, Branton D (2001) Voltage-driven DNA translocation through a nanopore. Phys Rev Lett 86:3435

168. Storm A, Storm C, Chen J, Zandbergen H, Joanny J-F, Dekker C (2005) Fast DNA translocation through a solid-state nanopore. Nano Lett 5:1193

169. Muthukumar M (2007) Mechanism of DNA transport through pores. Annu Rev Biophys Biomol Struct 36:435-450

170. Muthukumar M (2001) Translocation of a confined polymer through a hole. Phys Rev Lett 86:3188-3191

171. Muthukumar M (1999) Polymer translocation through a hole. J Chem Phys 111:10371
172. Izmitli A, Schwartz DC, Graham MD, de Pablo JJ (2008) The effect of hydrodynamic interactions on the dynamics of DNA translocation through pores. J Chem Phys 128:085102

173. Kantor Y, Kardar M (2004) Anomalous dynamics of forced translocation. Phys Rev E 69:021806

174. Gauthier MG, Slater GW (2008) Sequence effects on the forced translocation of heteropolymers through a small channel. J Chem Phys 128:175103

175. Luo KF, Metzler R (2010) Polymer translocation into a fluidic channel through a nanopore. Phys Rev E 82:021922

176. Bhattacharya S, Derrington IM, Pavlenok M, Niederweis M, Gundlach JH, Aksimentiev A (2012) Molecular dynamics study of MspA arginine mutants predicts slow DNA translocations and ion current blockades indicative of DNA sequence. ACS Nano 6:6960-6968

177. Li J, Zhang Y, Yang J, Bi K, Ni Z, Li D, Chen Y (2013) Molecular dynamics study of DNA translocation through graphene nanopores. Phys Rev E 87:062707

178. Luo K, Ala-Nissila T, Ying S-C, Metzler R (2009) Driven polymer translocation through nanopores: slow-vs.-fast dynamics. Europhys Lett 88:68006

179. Li-Zhen S, Meng-Bo L (2013) Study on the polymer translocation induced blockade ionic current inside a nanopore by Langevin dynamics simulation. J Phys: Condens Matter 25:465101

180. Tian P, Smith G (2003) Translocation of a polymer chain across a nanopore: a Brownian dynamics simulation study. J Chem Phys 119:11475

181. de Haan HW, Slater GW (2012) Memory effects during the unbiased translocation of a polymer through a nanopore. J Chem Phys $136: 154903$

182. Ikonen T, Bhattacharya A, Ala-Nissila T, Sung W (2012) Unifying model of driven polymer translocation. Phys Rev E 85:051803

183. Liu Z, Liu J, Xiao M, Wang R, Chen Y-L (2014) Conformationdependent translocation of a star polymer through a nanochannel. Biomicrofluidics 8:054107

184. He YD, Qian HJ, Lu ZY, Li ZS (2007) Polymer translocation through a nanopore in mesoscopic simulations. Polymer 48:36013606

185. Kapahnke F, Schmidt U, Heermann DW, Weiss M (2010) Polymer translocation through a nanopore: the effect of solvent conditions. J Chem Phys 132:164904

186. Duong-Hong D, Han J, Wang J, Hadjiconstantinou N, Chen Y, Liu G (2008) Realistic simulations of combined DNA electrophoretic flow and EOF in nano-fluidic devices. Electrophoresis 29:48804886

187. Feng JA, Ge XT, Shang YZ, Zhou LH, Liu HL, Hu Y (2011) Translocation of polymer through a nanopore studied by dissipative particle dynamics. Fluid Phase Equilib 302:2631

188. Guo JY, Li XJ, Liu Y, Liang HJ (2011) Translocation of polymers through a fluidic channel: a dissipative particle dynamics simulation study. J Chem Phys 134:8

189. Guo J, Li X, Liang H (2012) Dissipative particle dynamics simulation of fluid-driven polymer through a microchannel. Acta Polym Sin 2:160-167

190. Gennes P (1979) Scaling concepts in polymer physics. Cornell University Press, Ithaca

191. Li X, Pivkin IV, Liang H (2013) Hydrodynamic effects on flowinduced polymer translocation through a microfluidic channel. Polymer 54:4309-4317

192. Liu Z, Liu J, Xiao M, Wang R, Chen Y (2014) Conformationdependent translocation of a star polymer through a nanochannel. Biomicrofluidics 8:054107

193. Qiao R, He P (2007) Modulation of electroosmotic flow by neutral polymers. Langmuir 23:5810-5816

194. Pagonabarraga I, Rotenberg B, Frenkel D (2010) Recent advances in the modelling and simulation of electrokinetic effects: bridging 
the gap between atomistic and macroscopic descriptions. Phys Chem Chem Phys 12:9566-9580

195. Groot R (2003) Electrostatic interactions in dissipative particle dynamics simulation of polyelectrolytes and anionic surfactants. J Chem Phys 118:11265

196. Guo JY, Li XJ, Liu Y, Liang HJ (2012) Driven polymer translocation through a cylindrical nanochannel: interplay between the channel length and the chain length. Soft Matter 8:2769-2774

197. Beckersa J, Lowea C, De Leeuwa W (1998) An iterative PPPM method for simulating coulombic systems on distributed memory parallel computers. Mol Simul 20:369

198. Gonzalez-Melchor M, Mayoral E, Velazquez ME, Alejandre J (2006) Electrostatic interactions in dissipative particle dynamics using the Ewald sums. J Chem Phys 125:224107

199. Wang Y, Laaksonen A, Lu Z (2014) Implementation of nonuniform FFT based Ewald summation in dissipative particle dynamics method. J Comput Phys 235:666-682

200. Wang Y, Lu Z, Laaksonen A (2012) Specific binding structures of dendrimers on lipid bilayer membranes. Phys Chem Chem Phys 4:8348-8359

201. Chun B, Ladd AJC (2007) Interpolated boundary condition for lattice Boltzmann simulations of flows in narrow gaps. Phys Rev E 75:066705

202. Dünweg B, Ladd AJC (2008) Lattice Boltzmann simulations of soft matter systems. Adv Polym Sci 221:89

203. Ledesma-Aguilar R, Sakaue T, Yeomans JM (2012) Easier sieving through narrower pores: fluctuations and barrier crossing in flowdriven polymer translocation. Soft Matter 8:4306-4309

204. Ledesma-Aguilar R, Sakaue T, Yeomans JM (2012) Lengthdependent translocation of polymers through nanochannels. Soft Matter 8:1884-1892

205. Farahpour F, Maleknejad A, Varnik F, Ejtehadi MR (2013) Chain deformation in translocation phenomena. Soft Matter 9:27502759

206. Peskin CS (2002) The immersed boundary method. Acta Num $11: 1$

207. Ahlrichs P, Dünweg B (1998) Lattice Boltzmann simulation of polymer-solvent systems. Int J Mod Phys C 9:1429

208. Succi S (2001) The lattice Boltzmann equation for fluid dynamics and beyond, 1st edn. Oxford University Press, Oxford

209. Wolf-Gladrow D (2000) Lattice-gas cellular automata and lattice Boltzmann models: an introduction, 1st edn. Springer, New York

210. Aidun CK, Clausen JR (2010) Lattice-Boltzmann method for complex flows. Annu Rev Fluid Mech 42:439

211. Hsu C, Chen Y-L (2010) Microflow fractionation of deformable particles. J Chem Phys 133:034906

212. Chen Y-L (2014) Inertia- and deformation-driven migration of a soft particle in confined shear and Poiseuille flow. RSC Adv 4:17908

213. Ahlrichs P, Dünweg B (1999) Simulation of a single polymer chain in solution by combining lattice Boltzmann and molecular dynamics. J Chem Phys 111:8225

214. Chen Y-L, Lin Y-H, Chang J-F, Lin P-K (2014) Dynamics and conformation of semiflexible polymers in strong quasi-1D and2D confinement. Macromolecules 47:1199
215. Jo K, Chen YL, de Pablo JJ, Schwartz DC (2009) Elongation and migration of single DNA molecules in microchannels using oscillatory shear flows. Lab Chip 9:2348-2355

216. Adhikari R, Stratford K, Cates ME, Wagner AJ (2005) Fluctuating lattice Boltzmann. Europhys Lett 71:473

217. Succi S (2008) Lattice boltzmann across scales: from turbulence to DNA translocation. Eur Phys J 64:471-479

218. Reboux S, Capuani F, Gonzalez-Segredo N, Frenkel D (2006) Lattice-Boltzmann simulations of ionic current modulation by DNA translocation. J Chem Theory Comput 2:495-503

219. Melchionna S, Fyta MG, Kaxiras E, Succi S (2007) Exploring DNA translocation through a nanopore via a multiscale latticeBoltzmann molecular-dynamics methodology. Int J Mod Phys C 18:685-692

220. Fyta M, Sircar J, Kaxiras E (2008) Parallel multiscale modeling of biopolymer dynamics with hydrodynamic correlations. Int $\mathrm{J}$ Multiscale Com 6:25-37

221. Fyta M, Melchionna S, Bernaschi M, Kaxiras E, Succi S (2009) Numerical simulation of conformational variability in biopolymer translocation through wide nanopores. J Stat Mech Theor Exp 128:085102

222. Alapati S, Fernandes DV, Suh YK (2011) Numerical simulation of the electrophoretic transport of a biopolymer through a synthetic nano-pore. Mol Simul 37:466-477

223. Alapati S, Fernandes DV, Suh YK (2011) Numerical and theoretical study on the mechanism of biopolymer translocation process through a nano-pore. J Chem Phys 135:055103

224. Alapati S, Che WS, Suh YK (2013) Effect of Nanopore length on the translocation process of a biopolymer: numerical study. Materials 6:3989-4000

225. Cifra P, Benkova Z, Bleha T (2008) Effect of confinement on properties of stiff biological macromolecules. Faraday Discuss 139:377-392

226. Sakaue T, Yoshikawa K, Yoshimura S, Takeyasu K (2001) Histone core slips along DNA and prefers positioning at the chain end. Phys Rev Lett 87:078105

227. Symeonidis V, Karniadakis G, Caswell B (2005) Dissipative particle dynamics simulations of polymer chains: scaling laws and shearing response compared to DNA experiments. Phys Rev Lett 95:076001

228. Hoffman JF (2001) Questions for red blood cell physiologists to ponder in this millenium. Biol Blood Cells Mol Dis 27:57-61

229. Wan J, Ristenpart WD, Stone H (2008) Dynamics of shearinduced ATP release from red blood cells. Proc Natl Acad Sci USA 105:16432-16437

230. Lei H, Karniadakis GE (2012) Quantifying the rheological and hemodynamic characteristics of sickle cell anemia. Biophys J 102:185-194

231. Lei H, Karniadakis GE (2013) Probing vasoocclusion phenomena in sickle cell anemia via mesoscopic simulations. Proc Natl Acad Sci USA 110:11326-11330

232. Li X, Caswell B, Karniadakis GE (2012) Effect of chain chirality on the self-assembly of sickle hemoglobin. Biophys J 103:1130 1140 